Aus der Klinik für Hals-Nasen-Ohrenheilkunde

(Prof. Dr. med. D. Beutner)

der Medizinischen Fakultät der Universität Göttingen

\title{
Endoskopische Befunde nach Langzeitintubation
}

\author{
INAUGURAL-DISSERTATION \\ zur Erlangung des Doktorgrades \\ der Medizinischen Fakultät der \\ Georg-August-Universität zu Göttingen
}

vorgelegt von

Valeska Margarethe von Krosigk

aus

Hamburg

Göttingen 2019 
Dekan: $\quad$ Prof. Dr. rer. nat. H. K. Kroemer

Referent: $\quad$ Prof. Dr. med. A. Olthoff

Ko-Referent: $\quad$ PD Dr. med. S. Kazmaier

Drittreferent: $\quad$ Prof. Dr. med. M. Oppermann

Datum der mündlichen Prüfung: 21.04.2020 
Hiermit erkläre ich, die Dissertation mit dem Titel "Endoskopische Befunde nach Langzeitintubation" eigenständig angefertigt und keine anderen als die von mir angegebenen Quellen und Hilfsmittel verwendet zu haben.

Göttingen, den 01.04.2020

(Unterschrift) 


\section{Inhaltsverzeichnis}

Abbildungsverzeichnis .................................................................II

Tabellenverzeichnis ...................................................................II

Abkürzungsverzeichnis.............................................................. IV

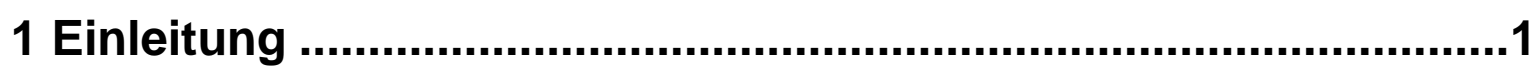

1.1 Historischer Hintergrund der Langzeitintubation ......................................

1.2 Laryngeale Komplikationen nach Langzeitintubation.................................

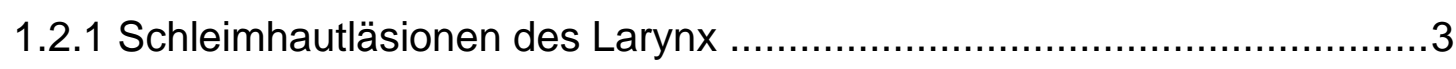

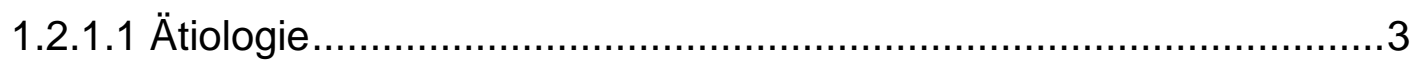

1.2.1.2 Prädilektionsstellen laryngealer Verletzungen ................................ 4

1.2.1.3 Akute Postintubationsschäden des Larynx ....................................

1.2.1.4 Chronische Larynxschäden nach Langzeitintubation .......................7

1.2.2 Bewegungsstörungen der Stimmlippen...........................................

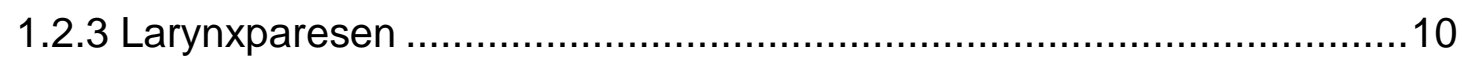

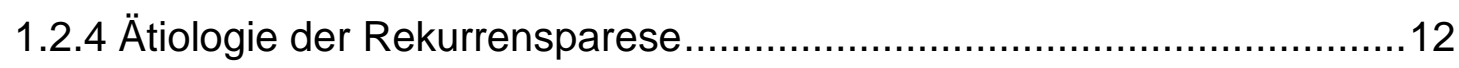

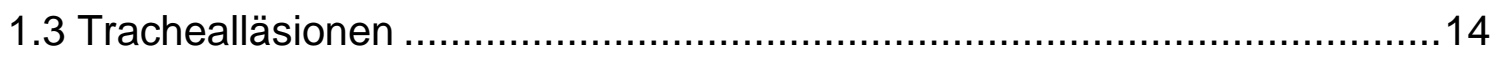

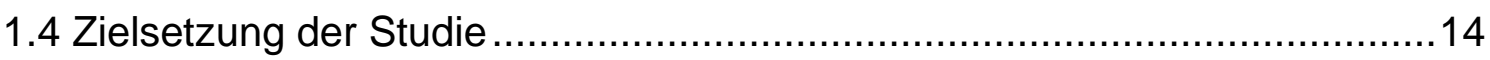

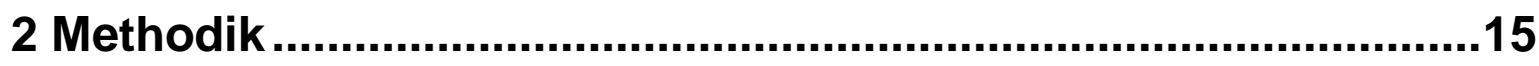

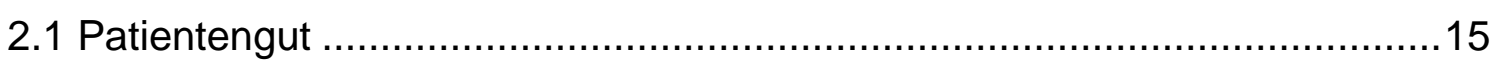

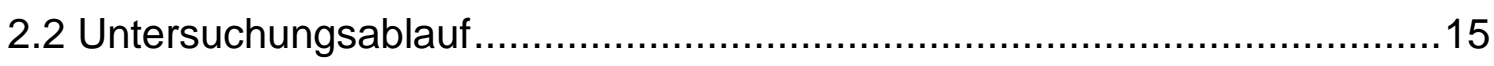

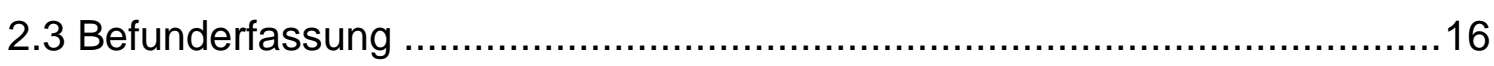

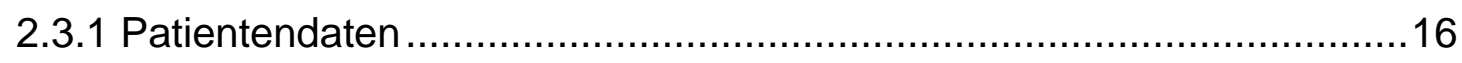

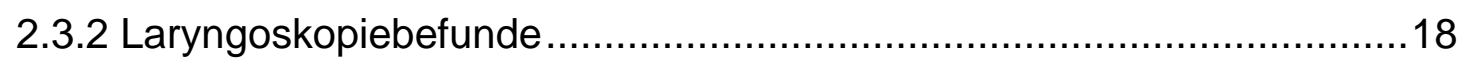

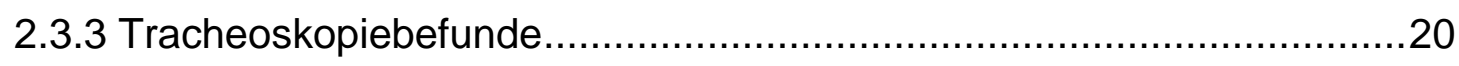


2.3.4 Qualitätsbeurteilung der Aufnahmen...............................................20

2.4 Zusammenfassung der Schleimhautbefunde ……………….................23

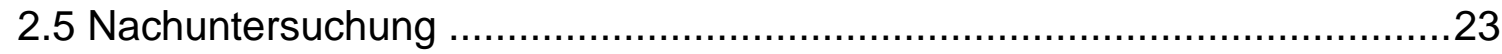

3 Ergebnisse ...........................................................................24

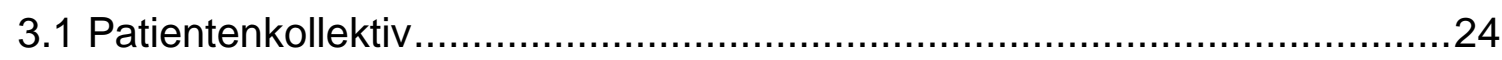

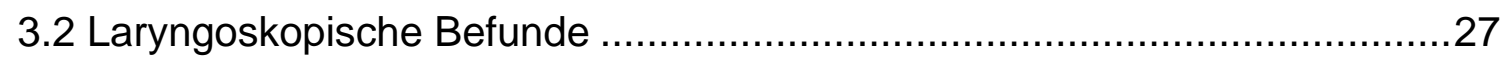

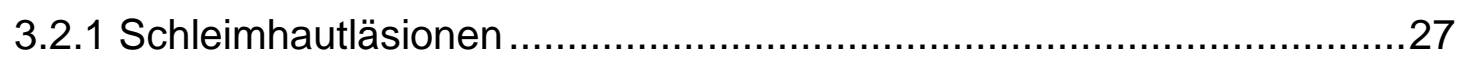

3.2.2 Bewegungsstörungen der Stimmlippen............................................30

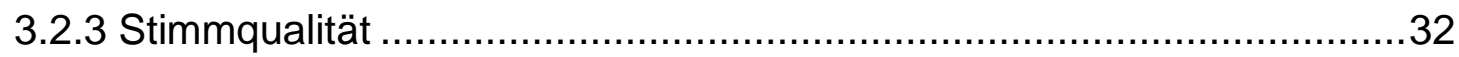

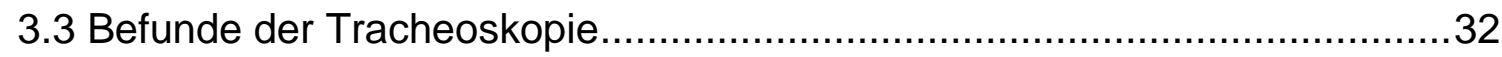

3.4 Qualitätsbeurteilung der Aufnahmen ......................................................33

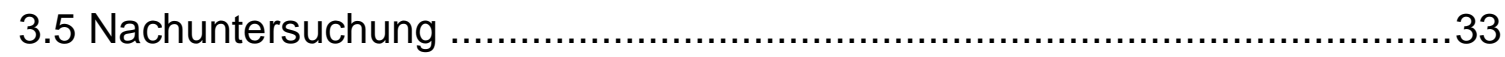

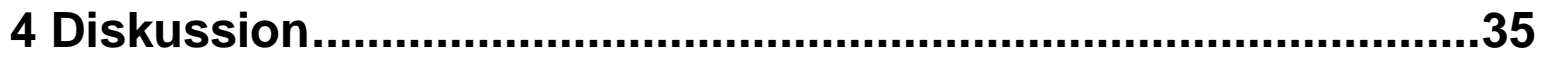

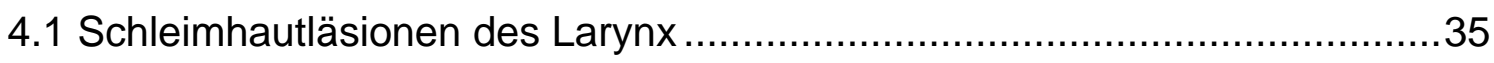

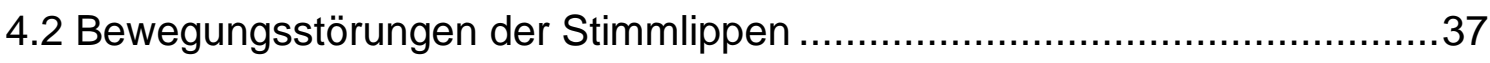

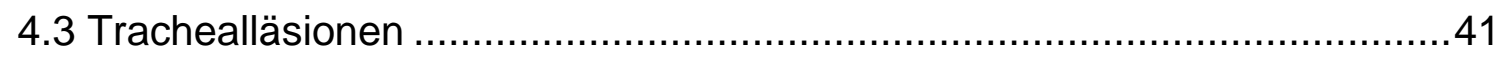

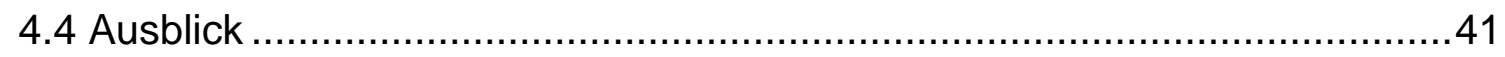

5 Zusammenfassung .............................................................43

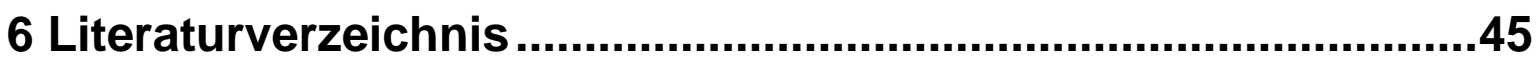




\section{Abbildungsverzeichnis}

Abbildung 1: Schematische Darstellung der Tubuslage im Larynx........................... 5

Abbildung 2: Kehlkopfpräparat ohne und mit Cuffdruck (Der Abdruck erfolgt mit freundlicher Genehmigung von Prof. Dr. A. Olthoff) ............................ 13

Abbildung 3: Dokumentationsbogen I zur Erfassung der endoskopischen Befunde

Abbildung 4: Dokumentationsbogen II zur Erfassung der endoskopischen Befunde, Untersuchungsaufnahmequalität, Grunderkrankung der Patienten und Stimmbewertung 22

Abbildung 5: $\quad$ Schleimhautbefunde der Laryngoskopie $(n=31)$ 27

Abbildung 6: Schleimhautbefunde der Laryngoskopie, nach Lokalisation geordnet

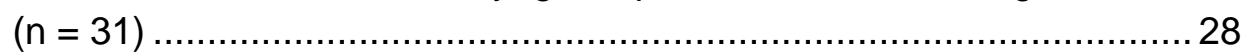

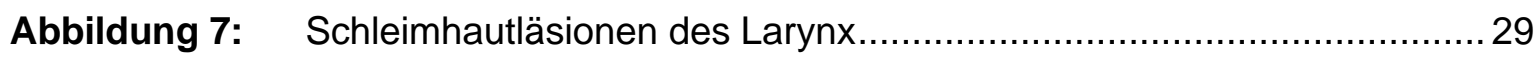

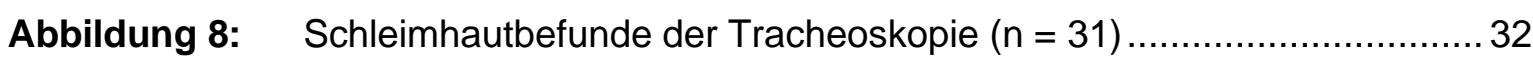

Abbildung 9: Trachealläsion nach $39 \mathrm{~h}$ Intubation (Pat.Nr. 19) .................................. 33

\section{Tabellenverzeichnis}

Tabelle 1: $\quad$ Bewertungsskala für Postintubationsschäden des Larynx ..................... 6

Tabelle 2: $\quad$ Ursachen einer Kehlkopflähmung …................................................ 12

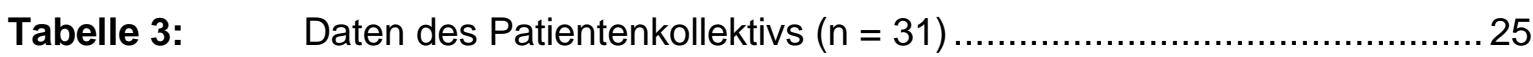

Tabelle 4: $\quad$ Zusammenfassung der Schleimhautbefunde unter Berücksichtigung von Intubationsdauer $(\mathrm{h})$ und Verletzungsausdehnung ......................2 29

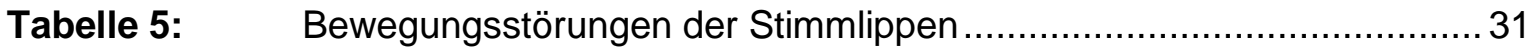




\section{Abkürzungsverzeichnis}

A.

ACVB

AK

AKE

CPAP

et al.

h

HNO

ICD

ITS

KHK

komb.

Li.

It.

M.

$\min$.

MKE

$\mathrm{Mm}$

$\mathrm{mmHG}$

N.

NAW

OP

OPS

$\mathrm{P}$

(p)

Pat.

pAVK

PVC

Re.

S.

s.
Arteria

Aortocoronarer-Venenbypass

Aortenklappe

Aortenklappenersatz

Continuous Positive Airway Pressure

et alii (Maskulinum), et aliae (Femininum) oder et alia (Neutrum) (lat.): und andere

hora (lat.): die Stunde

Hals-Nasen-Ohren-Heilkunde

International Classification of Diseases and Related Health Problems

Intensivstation

Koronare Herzkrankheit

kombiniert

Links

laut

Musculus

mindestens

Mitralklappenersatz

Musculi

Millimeter Quecksilbersäule

Nervus

Notarztwagen

Operation

Operationen und Prozedurenschlüssel

Portex-Tubus

paramedian

Patient

Periphere arterielle Verschlusskrankheit

Polyvinylchlorid

Rechts

siehe 
TF-Phonation Taschenfaltenphonation

V. Vena

V.a. Verdacht auf

WB Woodbridge-Tubus

Z.n. Zustand nach

ZVK Zentralvenöser Katheter 


\section{Einleitung}

Als Langzeitintubation wird eine Intubation über einen Zeitraum von mehr als 24 Stunden bezeichnet (Burns et al. 1979; Klose et al. 1978).

Heute wird die translaryngeale Intubation initial der Tracheotomie vorgezogen. Die einfachere Technik, die keinen operativen Eingriff erfordert, die geringere Komplikationsrate sowie ein geringeres Infektionsrisiko lassen die Intubation vorteilhafter erscheinen. Bei Beatmungen, die voraussichtlich länger als 21 Tage dauern, wird eine Tracheotomie empfohlen. Aktuell existiert jedoch keine einheitliche Regelung, nach welchem Zeitraum ein translaryngeal intubierter Patient tracheotomiert werden sollte. Empfohlen wird eine Tracheotomie zwischen dem fünften und siebten Intubationstag, um den Larynx zu schonen und die Komplikationsrate zu senken. Der Wechsel zur Trachealkanüle ermöglicht eine verminderte Analgosedierung des Patienten, eine Verkürzung der Intensivbehandlungszeit und eine Erleichterung der pflegerischen Maßnahmen (Brause und Friedrich 2011; Burns et al.1979; Freeman et al. 2005; Griffiths et al. 2005; Messingschlager 1970,).

Die vorliegende Arbeit sowie die ihr zugrunde liegende Studie befassen sich mit Schleimhautverletzungen des Larynx und der Trachea sowie mit Bewegungsstörungen der Stimmlippen nach Langzeitintubation. Einleitend wird ein Überblick über den historischen Hintergrund von Langzeitintubationen gegeben. Nachfolgend werden die Ätiologie und die Prädilektionsstellen intubationsbedingter Schleimhautverletzungen im Larynx und der Trachea sowie akute und chronische Postintubationsschäden beschrieben. Im Anschluss an einen Überblick über die Kehlkopfmuskulatur und deren Innervation werden die Bewegungsstörungen der Stimmlippen vorgestellt. Anschließend erfolgt die Betrachtung der Ätiologie einer Rekurrensparese gesondert.

Zum Abschluss der Einleitung wird die Zielsetzung der zugrunde liegenden Studie beschrieben.

\subsection{Historischer Hintergrund der Langzeitintubation}

Der arabische Arzt Avicenna (980-1037) führte vor ca. 1000 Jahren die erste orotracheale Intubation mit Metallrohren bei Erstickungszuständen durch. Bis Ende des 18. Jahrhunderts wurde die endotracheale Intubation ausschließlich zur Wiederbelebung Ertrunkener 
sowie zur Akutbehandlung Erstickender angewandt. Erste Versuche, spezielle Kanülen zur Intubation des Larynx zu entwerfen, stammen von Curry (1792), Leroy (1827) und Chaussier (1807). Keines dieser Geräte konnte sich jedoch in der praktischen Anwendung bei der Reanimation als Standard etablieren. Der Grund dafür waren allerdings hauptsächlich die Lungenverletzungen durch die damals genutzte Blasebalg-Beatmung, weniger tatsächliche Intubationsverletzungen (Luckhaupt und Brusis 1986).

Dem Pariser Chirurgen Desault (1738-1795) gelang es, erstmals erfolgreich Patienten mit Glottisödem durch Intubation zu behandeln. Dabei führte er blind Intubationen mit Magenschläuchen durch (Luckhaupt und Brusis 1986).

Die Diphterie-Epidemie in Europa und Nordamerika im 19. Jahrhundert machte die endotracheale Intubation nach der Methode von Trousseau und O`Dwyer zu einem konkurrenzfähigen Verfahren $\mathrm{zu}$ der bis dahin hauptsächlich durchgeführten Tracheotomie (Brandt 1986).

Die erste in der Literatur beschriebene orotracheale Intubationsnarkose mit anschließender Langzeitintubation wurde 1878 durch den Schotten Sir William MacEwen durchgeführt. Er ließ zwei Patienten mit Glottisödem nach lingualer Tumorresektion über mehr als 30 Stunden intubiert. MacEwen erweiterte damit das Anwendungsgebiet der Intubation auf die Anästhesie (Lindholm 1969; Messingschlager 1970).

Mit der Erfindung wichtiger Hilfsmittel wurde die endotracheale Intubation zwischen dem 19. und 20. Jahrhundert technisch verbessert. Um 1900 veröffentlichte Franz Kuhn erstmalig Ergebnisse naso- und orotrachealer Intubationen, die er mit einer selbst entwickelten, flexiblen Metallspiralsonde in großem Umfang durchgeführt hatte (Goerig und Brandt 1986). Alfred Kirstein ermöglichte 1895 mit der Entwicklung des ersten Laryngoskops die Intubation unter Sicht. Basierend auf weiteren technischen und medikamentösen Modifikationen wurde die endotracheale Intubation Mitte des 20. Jahrhunderts als Standardverfahren in der Anästhesie etabliert. (Luckhaupt und Brusis 1986; Brandt 1986).

Erste Einzelfallberichte zu Langzeitintubationen bei Erwachsenen lieferten Mitte des 20. Jahrhunderts Gillespie, Foregger und Urry. 1951 beschrieb Nilsson Langzeitintubationen bei Barbituratvergiftungen. Dam und Zwergius veröffentlichten 1952 erste Berichte über den Einsatz von Langzeitintubationen im Rahmen maschineller Beatmungen nach thorakalen Eingriffen (Lindholm 1969; Roth et al. 1967; Young und Steward 1953). 
Die Entwicklung gewebeschonender und thermoplastischer Kunststoffe in Verbindung mit zunehmender Pflegeerfahrung und immensem technischen Fortschritt in der Intensivmedizin haben die Langzeitintubation zu einem häufig angewandten Verfahren gemacht (Klose et al. 1978; Mantel und Westhues 1967). Die dabei auftretenden Komplikationen und Folgen für Larynx und Trachea wurden seither in diversen Studien evaluiert, welche im Folgenden aufgeführt werden.

\subsection{Laryngeale Komplikationen nach Langzeitintubation}

Pharyngolaryngeale Beschwerden gehören neben Schmerzen und systemischen Komplikationen, wie beispielsweise Pneumonien, zu den häufigsten Symptomen nach Intubationen. Vielfach beschrieben sind Heiserkeit, Dyspnoe, Dysphagie und Aspiration, deren Auftreten mit steigender Intubationsdauer deutlich zunimmt (Burns et al. 1979; Ding et al. 2006).

Schleimhautläsionen des Larynx und Bewegungsstörungen der Stimmlippen werden als ursächlich für diese Symptome angenommen (Bishop et al. 1984; Vila et al. 1997).

\subsubsection{Schleimhautläsionen des Larynx}

\subsubsection{1 Ätiologie}

Erschwerte Sichtverhältnisse oder eine unvorsichtige Vorgehensweise können bereits bei der Intubation zu traumatisch-mechanischen Schäden der Kehlkopfschleimhaut führen (Larsen 2013). Nach längerer Intubationsdauer sind Schleimhautverletzungen des Kehlkopfes eine vielfach beschriebene Erscheinung. Die Häufung von Läsionen an bestimmten Kehlkopfstrukturen ist insbesondere durch die Anatomie des Kehlkopfes bedingt (Benjamin und Holinger 2008; Bishop et al. 1984; Colice 1992; Ellis und Bennett 1977; Lemburg et al. 1971).

$\mathrm{Zu}$ den Einflussfaktoren gehören Bewegungen des Tubus und das Material der Tubusoberfläche. Die Größe des verwendeten Tubus wird als sehr relevant bewertet (Burns et al. 1979; Harrison und Tonkin 1968). Bei Frauen treten Schleimhautläsionen aufgrund der schmaleren Anatomie des Kehlkopfes deutlich häufiger auf (Bishop et al. 1984). Besondere Bedeutung kommt daher der Wahl der richtigen Tubusgröße im Verhältnis zur Kehlkopfanatomie zu. Die Tubusgröße wird anhand des Patientenalters festgelegt. Im Allgemeinen gilt, dass zu kleine Tuben zu einem erhöhten Widerstand gegen die 
Strömung der Atemluft im Tubus führen und dass zu groß gewählte Tuben Larynx und Trachea schädigen (Larsen 2013). Zu kleine Tuben können ebenfalls durch den zur sicheren Blockung erforderlichen hohen Cuffdruck zu Schleimhautschädigungen führen. Stand der Technik sind mittlerweile Tuben aus Polyvinylchlorid (PVC) mit weichen Cuffs aus Silikon. Bei Erwärmung auf Körpertemperatur passt sich die Tubusform der Anatomie des oberen Respirationstraktes an. Schleimhautirritationen sollen hierdurch reduziert werden (Larsen 2013; Pfannenstiel et al. 2007).

Um die Bewegung zwischen Larynx und Tubus so gering wie möglich zu halten, werden flexible Verbindungsstücke zwischen Tubus und Beatmungsmaschine eingesetzt und der Tubus am Patienten mittels Pflasterstreifen fixiert (Klose et al. 1978).

Infektionen, Steroidmedikation, systemische Erkrankungen wie Diabetes mellitus und der laryngotracheale Reflux werden ebenfalls als Faktoren diskutiert, die die Entstehung laryngealer Verletzungen begünstigen (Benjamin und Holinger 2008).

\subsubsection{Prädilektionsstellen laryngealer Verletzungen}

Die vordere Wand des hyalinen Knorpelgerüstes des Larynx bildet der Schildknorpel (Cartilago thyroidea). Die hintere Wand besteht aus den Aryknorpeln (Cartilago arytenoidea) und dem Ringknorpel (Cartilago cricoidea). Die Seitenwände werden durch den Ringknorpel und die nach ventral schmal zulaufenden Anteile des Schildknorpels gebildet.

Die Öffnungsform der Glottis wird von den Ligamenti vocales bestimmt, die ventral am Schildknorpel und dorsal an den Processi vocales der Aryknorpel ansetzen. Durch die an den Processi muscularis der Aryknorpel ansetzende Muskulatur kann die Form und Größe der Glottisöffnung durch Drehung der Aryknorpel um ihre Längsachse verändert werden.

So entsteht die unregelmäßige fünfeckige Struktur des inneren Kehlkopfes, gebildet durch den Ringknorpel, die Processi vocales der Aryknorpel und die Stimmlippen (Bishop et al. 1984; Probst et al. 2008).

Die Kehlkopfanatomie bedingt eine dorsale Lage des Tubus. Daher ist insbesondere die Schleimhaut im hinteren Bereich der Glottis häufig von Schleimhautverletzungen betroffen (s. Abbildung 1). Die schmale Auflagefläche des Tubus kann dort zu hohen Druckbelastungen führen. Weymuller et al. (1983) detektierten in einem Hundeversuch Drücke im Bereich der Aryknorpel von bis zu 400 mmHg. Dieser Wert liegt deutlich 
oberhalb des kapillären Perfusionsdruckes der Schleimhaut von ungefähr $30 \mathrm{mmHg}$. Hohe Drücke können ischämische Schäden der Schleimhaut hervorrufen (Weymuller et al. 1983). Gehäuft treten Schleimhautläsionen an der hinteren Kommissur, den Processi vocales der Aryknorpel, dem hinteren Stimmlippenbereich und der Interarytenoidregion auf (Benjamin und Holinger 2008; Colice 1992; Lindholm 1969; Stauffer et al. 1981; Vila et al. 1997; Whited 1979).

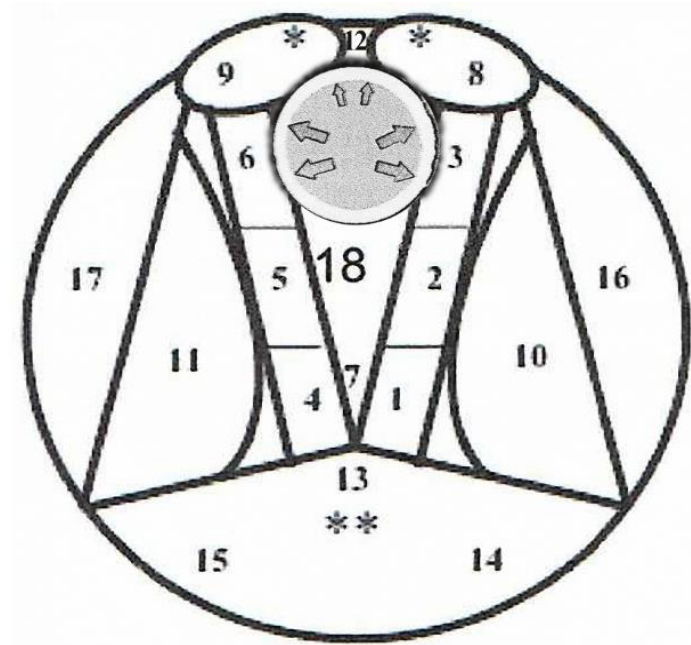

\section{Legende:}

1 vordere Glottis links

2 mittlere Glottis links

3 hintere Glottis links

4 vordere Glottis rechts

5 mittlere Glottis rechts

6 hintere Glottis rechts

7 vordere Kommissur

8 Aryknorpel links

18 Ringknorpel

** präepiglottischer Raum
9 Aryknorpel rechts 10 supraglottisch links 11 supraglottisch rechts 12 Interaryregion 13 Petiolus

14 Epiglottis links

15 Epiglottis rechts

16 Sinus piriformis links

17 Sinus piriformis rechts

* postcricoidal

Abbildung 1: Schematische Darstellung der Tubuslage im Larynx (Quelle: Eigene Darstellung)

Der Cricoidknorpel bildet eine subglottische Engstelle. Aufgrund seiner ringförmigen und absolut rigiden Form finden sich hier häufig Läsionen. Der Tubus muss sich an dieser Stelle der Anatomie entsprechend umformen. Gemessen in einem Hasenversuch kann dies zu Druckbelastungen der Ringknorpelschleimhaut von bis zu $90 \mathrm{mmHg}$ führen (Stenqvist und Bagge 1979; Stenqvist et al. 1979). Überwiegend treten die Schleimhautschäden dort latero-posterior auf (Lindholm 1969). 


\subsubsection{Akute Postintubationsschäden des Larynx}

Ödeme, Hämatome, Hyperämien, Ulzerationen und Erosionen sind häufig vorkommende akute Schleimhautschäden nach endotrachealen Langzeitintubationen (Tubusliegezeit > 24 Stunden) (Burns et al. 1979; Lindholm 1969; Vila et al. 1997).

Das Auftreten erster laryngealer Schleimhautläsionen ist in der Literatur bereits nach einer Intubationsdauer von drei bis sieben Stunden beschrieben (Stauffer et al. 1981).

Zum Schweregrad auftretender Läsionen veröffentlichte Lindholm (1969) Studienergebnisse von 125 Erwachsenen und 36 Kindern nach Langzeitintubation (min. 24 h), bei denen er laryngeale und tracheale Schleimhautbefunde nach der Extubation beschrieb. Diese Untersuchungen dienten dabei als Basis für eine Bewertungsskala, in der die auftretenden Läsionen in Schweregrade eingeteilt wurden. In Tabelle 1 sind die Untersuchungsergebnisse des Larynx anhand der Bewertungsskala zusammengefasst. Sämtliche untersuchten Patienten erlitten Mukosaverletzungen. Am häufigsten traten Verletzungen II. Grades auf.

Lindholm (1969) beschrieb einen Zusammenhang zwischen der Häufigkeit laryngealer Verletzungen und der Intubationsdauer. Eine Korrelation des Schweregrades der Verletzungen mit der Intubationsdauer konnte nicht festgestellt werden.

Tabelle 1: $\quad$ Bewertungsskala für Postintubationsschäden des Larynx (nach Lindholm 1969)

\begin{tabular}{|l|l|l|}
\hline Schweregrade & $\begin{array}{l}\text { Symptome } \\
\text { Grad I }\end{array}$ & $\begin{array}{l}\text { Betroffene } \\
\text { Patienten } \\
\text { in \% }\end{array}$ \\
Grad II: & $\begin{array}{l}\text { Hyperämien, Ödeme ohne makroskopisch } \\
\text { sichtbare Ulzeration } \\
\text { Ulzerationen, }<1 / 3 \text { des Atemwegsumfangs } \\
\text { umfassend } \\
\text { tiefe Ulzerationen }<1 / 3 \text { des Atemwegsumfangs } \\
\text { oder oberflächliche Ulzerationen }>1 / 3 \text { des } \\
\text { Atemwegsumfangs umfassend } \\
\text { tiefe Mukosaulzerationen mit freiliegendem } \\
\text { Knorpel oder tiefe Ulzerationen mehr als } 1 / 3 \text { des } \\
\text { Atemwegsumfangs umfassend }\end{array}$ & $\mathbf{2 2}$ \\
Grad IV: & $\mathbf{1 8}$ \\
\hline
\end{tabular}


Burns et al. (1979) unterschieden bei 117 Patienten Schleimhautveränderungen mit intakter Mukosa und Mukosa durchdringenden Verletzungen. Das Patientenkollektiv wurde in vier Gruppen eingeteilt. Patienten mit einer Intubationsdauer von 0 bis 48 Stunden (Gruppe 1), 49 bis 72 Stunden (Gruppe 2), 73 bis 120 Stunden (Gruppe 3) und mehr als 120 Stunden (Gruppe 4). Im Ergebnis wurde eine steigende Inzidenz beider Schädigungsformen mit zunehmender Intubationsdauer detektiert (Burns et al. 1979).

Stauffer et al. (1981) stellten in ihren Untersuchungen keine Korrelation zwischen Intubationsdauer und laryngealen Verletzungen fest. Jedoch fanden sie ein vermehrtes Auftreten laryngealer Verletzungen nach orotrachealer Intubation im Vergleich zu nasotrachealer Intubation (Stauffer et al. 1981).

In einer prospektiven Studie endoskopierten Colton House et al. (2011) Patienten mit einer Intubationsdauer von min. 48 Stunden in den ersten 24 Stunden nach Extubation. Sämtliche 61 untersuchten Patienten wiesen laryngeale Verletzungen auf. Eine Korrelation der verwendeten Tubusgröße mit dem Schweregrad auftretender laryngealer Verletzungen und der Intubationsdauer konnte jedoch nicht festgestellt werden (Colton House et al. 2011).

Hämatome der Stimmlippen sind hauptsächlich Intubationstraumata. Die linke Stimmlippe ist dabei besonders häufig betroffen, meist verursacht durch die Tubusführung mit der rechten Hand (Benjamin und Holinger 2008; Reber et al. 2007).

\subsubsection{Chronische Larynxschäden nach Langzeitintubation}

Bleibende Schäden des Larynx, wie Intubationsgranulome, Granulationspolypen oder die schwerwiegende Komplikation einer Stenose, treten nur selten auf. Stenosen entstehen meist erst durch sekundäre Schleimhautheilung (Colice 1992). McGovern (1971) berichtete über das Auftreten laryngealer Stenosen erst Wochen nach der Extubation (Bishop et al. 1984). In der Literatur wird die Häufigkeit auftretender Larynxstenosen mit weniger als 0,5\% angegeben (Colice 1992; Colice et al. 1989).

Eine Korrelation der Intubationsdauer mit dem Auftreten laryngotrachealer Stenosen stellte Whited (1979) in einer prospektiven Studie mit insgesamt 200 Patienten fest. Nach 2 bis 5 Intubationstagen traten bei $2 \%$ der Patienten im Verlauf chronische Stenosen auf, nach 6 bis 10 Tagen Intubationsdauer bei $5 \%$ der Untersuchten und nach einer Intubationsdauer von 11 bis 24 Tagen bei $12 \%$ der Patienten. Besonders häufig waren 
gemäß der Studie allerdings Patienten betroffen, die nach Langzeitintubation tracheotomiert wurden.

Bei den meisten Patienten bilden sich laryngeale Läsionen von Schleimhäuten bereits wenige Wochen nach der Extubation vollständig durch die primäre Wundheilung zurück (Colice 1992; Lindholm 1969). Colice (1992) endoskopierte 51 Patienten in einer Followup Studie zweiwöchentlich nach der Extubation bis zur vollständigen Regeneration der Schleimhäute. In $78 \%$ der Fälle heilten die Läsionen in den ersten acht Wochen nach der Extubation vollständig aus. In der Analyse von Lindholm (1969) kam es zur Restitutio ad integrum bei $61 \%$ der Patienten.

\subsubsection{Bewegungsstörungen der Stimmlippen}

Kehlkopfinnervation: Der Nervus (N.) vagus ist über den N. laryngeus superior und N. laryngeus inferior (= N. recurrens) sowohl für die motorische als auch für die sensible und sensorische Innervation des Larynx und der Trachea verantwortlich (Probst et al. 2008). Unterhalb des Ganglion inferius zweigen vom N. vagus die Fasern des N. laryngeus superior ab. Letzterer verläuft lateral der Arteria (A.) carotis interna, die er etwa in Höhe des vierten Halswirbels oberhalb des Abganges aus der A. carotis communis kreuzt. Hiernach teilt er sich in den Ramus internus und externus.

Der Ramus externus zieht auf dem Musculus (M.) constrictor pharyngis inferior zum M. cricothyroideus, dem einzigen außen am Kehlkopf anliegenden Muskel, den er motorisch versorgt (Kruse et al. 2006; Olthoff et al. 2003; Sobotta 2010). Der Ramus internus zieht gemeinsam mit A. und Vena (V.) laryngea superior durch die Membrana thyrohyoidea in das Innere des Larynx und versorgt sensibel die Schleimhaut der kranialen Kehlkopfanteile einschließlich der Stimmlippenebene (Probst et al. 2008; Prometheus 2012). Zudem werden afferente propriozeptive Fasern im Ramus internus angenommen, welche von den Muskelspindeln der Larynxmuskulatur gespeist werden (Olthoff et al. 2003).

Der N. laryngeus recurrens verläuft seitendifferent. Nach Abgang aus dem N. vagus im unteren Halsbereich tritt er in den Brustraum ein. Dort umschlingt der linke N. recurrens den Aortenbogen und der rechte die A. subclavia (bzw. Truncus brachiocephalicus). Der weitere kraniale Verlauf zwischen Trachea und Ösophagus mit Überkreuzen der A. thyroidea dorsal der Schilddrüse bis zum Eintritt in das Kehlkopfinnere ist seitengleich. Der N. recurrens gibt feine Äste an die Unterseite des M. cricoarytaenoideus posterior ab und zieht dann hinter dem Cornu inferius des Schildknorpels kranial um das 
Cricothyroidgelenk in das Innere des Kehlkopfes. Dieser Ast wird als Ramus anterior des N. recurrens bezeichnet. Im Inneren des Kehlkopfes folgen weitere Verzweigungen zum M. thyroarytaenoideus internus (M. vocalis) und externus sowie zum $M$. cricoarytaenoideus lateralis (Probst et al. 2008; Prometheus 2012; Sobotta 2010). Der Ramus posterior zieht zum M. interarytaenoideus (Schiel et al. 2004).

Der Ramus anterior und ist aufgrund seines anatomischen Verlaufes durch einen liegenden Tubus und insbesondere durch den Druck des Tubuscuff besonders gefährdet. Ihm gilt im Rahmen dieser Studie besondere Aufmerksamkeit.

Der N. laryngeus recurrens versorgt sensibel die Larynxschleimhaut unterhalb des Glottisspaltes sowie die Trachealschleimhaut über feine Äste. Motorisch innerviert der N. laryngeus recurrens die gesamte endolaryngeale Muskulatur (Prometheus 2012).

Kehlkopfmuskulatur: Bei der Kehlkopfmuskulatur werden innere und äußere Anteile unterschieden. Die innere Kehlkopfmuskulatur ist für die Bewegung und den Tonus der Stimmlippen zuständig und die äußere für die Hebung und Senkung des gesamten Kehlkopfes. Obwohl dem Kehlkopf außen und vorn anliegend wird der M. cricothyroideus aufgrund seiner Funktion den inneren Kehlkopfmuskeln zugerechnet. Er wird in der Praxis auch als Anticus bezeichnet. Im Gegensatz zur übrigen Kehlkopfmuskulatur wird der M. cricothyroideus durch den N. laryngeus superior innerviert (Prometheus 2012).

Die weitere innere Kehlkopfmuskulatur besteht aus den $\mathrm{Mm}$. cricoarytaenoideus posterior und lateralis, dem M. thyroarytaenoideus internus (M. vocalis) und externus sowie den Mm. interarytaenoideus transversus und obliquus (Sobotta 2010).

Funktionell bilden M. vocalis und M. cricothyroideus die Einheit der Stimmlippenspanner. Der M. cricoarytaenoideus lateralis wird als Phonationsmuskel bezeichnet. Durch das Öffnen des Pars intercartilaginea bei gleichzeitigem Verschluss des Pars intermembranacea wird durch Annäherung der Spitzen der Processi vocalis die Phonation eingeleitet.

Die Gruppe weiterer Stimmlippenadduktoren bestehen aus dem M. vocalis, M. interarytaenoideus transversus und obliquus sowie dem M. thyroarytaenoideus externus. Der einzige Stimmlippenöffner ist der M. cricoarytaenoideus posterior (Schiebler et al. 1999). 


\subsubsection{Larynxparesen}

Die häufigste Larynxparese ist die des N. laryngeus inferior. Die auch als Rekurrensparese bezeichnete Parese führt zu laryngoskopisch gut darstellbaren Bewegungsstörungen der Stimmlippen. Im Kehlkopfbereich wird der Begriff „Parese“ sowohl für unvollständige als auch für vollständige Lähmungen verwandt. Klinisch zeigen sich jedoch Unterschiede.

Nach der in Lehrbüchern noch gültigen „Wagner-Grossmann-Theorie“ führt eine komplette Lähmung des N. laryngeus inferior zu einem Ausfall der vom N. recurrens versorgten inneren Kehlkopfmuskulatur. Der Tonus des vom oberen Kehlkopfnerven versorgten $M$. cricothyroideus bleibt intakt und verursacht so bei Phonation und Inspiration einen straffen Stillstand der ipsilateralen Stimmlippe nahe der Mittellinie in der sogenannten Paramedianstellung. Bei einem zusätzlichen Ausfall des N. laryngeus superior zeigt sich meist eine schlaffe Lähmung in Intermediärposition (Eckel und Sittel 2001; Schulte und Seidner 2005; Wendler und Seidner 2005).

Nicht selten ist zusätzlich eine adduktorische Restbeweglichkeit der Stimmlippen zu beobachten, die als Folge einer unvollständigen Rekurrensparese angesehen wird. Ein funktionelles Überwiegen der Adduktoren gegenüber den Glottisöffnern bedingt hierbei die Paramedianstellung. Jedoch lassen sich nicht sämtliche beobachteten Bewegungsstörungen mit dieser Theorie erklären (Heinemann und Klaff 1982). In der Literatur werden immer wieder auch Intermediärpositionen beschrieben (Hahn et al. 1970).

Ein sicherer Rückschluss auf den Läsionsort anhand der Stimmlippenstellung ist nicht möglich. Der Versuch einer Kategorisierung (Dralle et al. 2004) gelang bisher nicht zufriedenstellend.

Mehrere Studien an Kehlkopfpräparaten belegten eine große Variationsbreite der endolaryngealen Aufzweigung des N. laryngeus inferior (Brandwein et al. 1986; Sunderland und Swaney 1952). Die Theorie einer strikten Aufteilung in einen anterolateralen adduktorischen und einen postero-medialen abduktorischen Ast mit jeweiliger Innervation einzelner Kehlkopfmuskeln ist auch elektromyographisch nicht belegbar (Arold 1981).

Die klinische Leitsymptomatik einer einseitigen Rekurrensparese ist die Heiserkeit, die insbesondere bei lautem Sprechen auftritt. Patienten beklagen darüber hinaus häufig eine phonatorische Dyspnoe, die durch das Entweichen unmodulierter Luft aufgrund der 
Schlussinsuffizienz bei Phonation ausgelöst wird (Eckel und Sittel 2001; Schulte und Seidner 2005; Wendler und Seidner 2005; Zumtobel et al. 2006).

Stroboskopisch schwingt die betroffene Stimmlippe durch den Tonusverlust des M. vocalis als homogene Masse mit geringer Schwingungsamplitude und mangelnder Randkantenverschiebung. Bei einer Restbeweglichkeit in Paramedian- oder Medianstellung ist eine Schwingungssynchronisation durch Berührung der gesunden Stimmlippe möglich. In Intermediärposition schwingt die gelähmte Stimmlippe unabhängig von der gesunden Seite.

Das Leitsymptom der doppelseitigen Rekurrensparese in Paramedianstellung ist die Atemnot. Bei zusätzlicher Schleimhautschwellung oder einem laryngealen Infekt kann ein doppelseitiger Stimmlippenstillstand zu lebensbedrohlichen Komplikationen führen (Wendler und Seidner 2005).

Auch zerebrale Schädigungen können ursächlich für Bewegungsstörungen der Stimmlippen sein. Eine einseitige Einschränkung der Stimmlippenmobilität ist dabei jedoch nicht zu beobachten, weil die kortikale Repräsentation der laryngealen Sensomotorik bilateral ist. Häufiger treten Symptomkomplexe mit Dysarthrophonien, Sprechapraxie und Aphasie auf, teilweise mit Störung der Phonations- oder Sprechatmung. Hirnstammläsionen können hingegen zum Bild einer peripheren (Vagus-) Lähmung führen.

Läsionen des N. vagus führen je nach Lokalisation zu unterschiedlichen Lähmungsbildern, die meist durch eine kardiale Symptomatik begleitet sind (Wendler und Seidner 2005).

Ein isolierter Ausfall des N. laryngeus superior ist sehr selten. Klinisch wird diese Lähmung relevant, wenn der Ramus internus beteiligt ist, was zu einer Störung des Schluckaktes mit Aspiration führen kann (Probst et al. 2008; Wendler und Seidner 2005). Mit Bezug auf die Stimme kann eine eingeschränkte Modulationsfähigkeit zu höheren Frequenzen beobachtet werden. 


\subsection{4 Ätiologie der Rekurrensparese}

In Tabelle 2 wird eine Übersicht über die Ätiologie der peripheren Rekurrensparese gegeben. Am häufigsten tritt eine periphere Rekurrenslähmung nach Strumektomie auf (0,3-3 \%) (Endo et al. 2007; Kroll et al. 1990; Wendler und Seidner 2005).

Tabelle 2: $\quad$ Ursachen einer Kehlkopflähmung (Quelle: eigene Entwicklung)

\begin{tabular}{|l|}
\hline Ursachen einer Kehlkopflähmung \\
Strumektomie \\
Grippeneuritis \\
Hals- und Thoraxtraumen \\
Operationen an Hals und Thorax \\
Herz- und Gefäßerweiterungen (hauptsächlich Aorta) \\
Benigne und maligne Struma \\
Raumfordernde Prozesse im Ösophagus \\
Infektionserkrankungen: Lyme-Borreliose, Lues, Mononukleose \\
Diabetische (Poly-) Neuropathie \\
Intubation \\
Intoxikation
\end{tabular}

Die Inzidenz intubationsbedingter Rekurrensparesen beträgt laut Friedrich et al. (2000) und Friedrich (2002) 1,4\% nach Kurzzeitintubation. In einer prospektiven Studie von Colton House et al. (2011) wurden nebenbefundlich bei $39 \%$ der untersuchten Patienten nach Langzeitintubation (min. $48 \mathrm{~h}$ ) Bewegungsstörungen der Stimmlippen dokumentiert.

Die Schädigungsmechanismen im Rahmen einer Intubation sind multifaktoriell und werden in der Literatur kontrovers diskutiert.

Ein Mechanismus ist die Schädigung des endolaryngeal verlaufenden Astes des N. laryngeus recurrens durch einen zu hoch sitzenden Tubuscuff. Erstmalig durch Hahn et al. (1970) vermutet, gilt dieser Mechanismus nach zahlreichen anatomischen Studien (Cavo 1985; Ellis und Pallister 1975; Olthoff et al. 2003) als wahrscheinlichste Ursache einer Stimmlippenlähmung nach Intubation (Baraka et al. 1981; Lu et al. 1999; Wason et al. 2004). Der Tubuscuff drückt dabei den paraglottischen Raum gegen die 
Schildknorpelplatte und führt so zu einer Druckbelastung des Ramus anterior des N. recurrens.

Abbildung 2 zeigt ein Kehlkopfpräparat umschlossen von einer Nachformung des Schildknorpels aus Kunststoff mit und ohne Cuffdruck. Die Kompression des Ramus anterior des N. recurrens ist hierbei nachvollziehbar dargestellt.
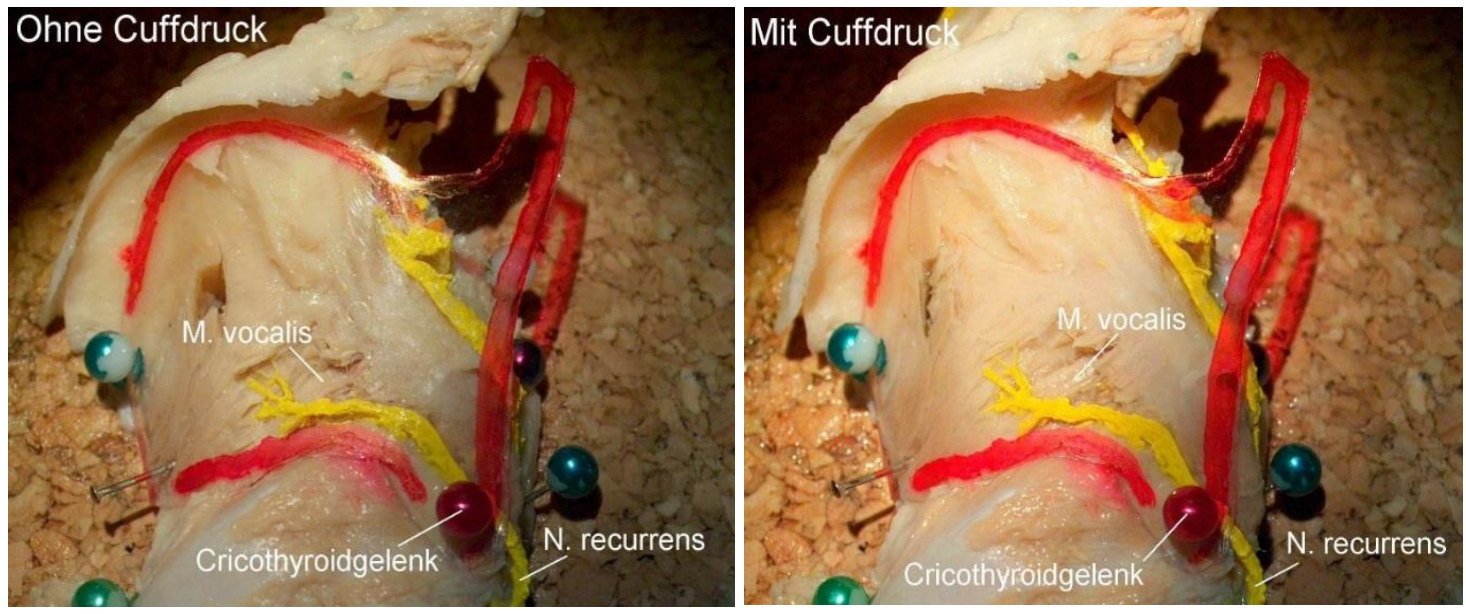

Abbildung 2: Kehlkopfpräparat ohne und mit Cuffdruck

(Der Abdruck erfolgt mit freundlicher Genehmigung von Prof. Dr. A. Olthoff)

Brandwein et al. (1986) stellten in acht Kehlkopfpräparaten den Verlauf des N. recurrens dar und vermuteten eine Schädigung durch Druckbelastung des Nerven bei hoch sitzendem Tubuscuff zwischen den Arytenoidknorpeln und der Lamina thyroidea.

Aber auch ein zu stark aufgeblasener Cuff kann zu einer Schädigung des N. recurrens führen (Cox und Welborn 1981). Laut Minuck (1976) kann es intraoperativ zu einem Anstieg des Cuffdrucks kommen, wenn Narkosegas und Sauerstoff schneller in den Cuff einströmen, als Stickstoff ausströmt.

Bauer (1958) sah eine Dehnungsverletzung des Nerven als ursächlich für Rekurrensparesen an. Er vermutete, dass es durch die Erschlaffung des Muskeltonus und den Ausfall der Schutzreflexe infolge der Relaxantiengabe bei Intubation zu einer Überstreckung und Zerrung der Halsweichteile bei Lagerung und Bewegung des Patienten kommt. 


\subsection{Trachealläsionen}

Verletzungen der Trachea sind ähnlich denen im Larynx. Meist sind sie auf Höhe des Cuffs und an der Kontaktstelle zwischen der Tubusspitze mit der Trachealwand lokalisiert (Keane et al. 1982; Lindholm 1969; Stauffer et al. 1981).

Die verminderte Schleimhautperfusion, die dadurch entstehende Hypoxie durch den Cuffdruck sowie die mechanische Verletzung der Schleimhaut durch die Tubusspitze sind nachgewiesene Ursachen auftretender Läsionen (Cooper und Grillo 1972).

\subsection{Zielsetzung der Studie}

Laryngeale Bewegungsstörungen sind aus der Literatur bekannte Komplikationen nach Kurzzeitintubation, wie zum Beispiel nach einer Intubationsnarkose mit weniger als 24 Stunden Intubationsdauer.

Wie häufig und mit welchem Schweregrad Bewegungsstörungen nach Langzeitintubation auftreten, ist bisher jedoch nicht belegt und soll daher durch die Endoskopie von Patienten nach einer Mindestintubationsdauer von 24 Stunden untersucht werden.

Endoskopische Befunde der Larynxschleimhaut nach Langzeitintubation sind in der Literatur ausgiebig dargestellt. Zu einer möglichen Korrelation zwischen Intubationsdauer und Häufigkeit sowie Schweregrad auftretender Larynxverletzungen gibt es jedoch divergierende Ergebnisse.

Daher wird zusätzlich der mögliche Zusammenhang zwischen Intubationsdauer und Häufigkeit sowie Schweregrad auftretender Schleimhautverletzungen untersucht. 


\section{Methodik}

Im folgenden Kapitel wird die Methodik der prospektiven Studie, die dieser Arbeit zugrunde liegt, im Detail vorgestellt. Dazu wird zunächst das Patientengut der Studie beschrieben sowie der genaue Untersuchungsablauf dargestellt. Im Weiteren wird die Befunderfassung der Laryngoskopie und Tracheoskopie anhand von zwei standardisierten Dokumentationsbögen sowie die Qualitätsbeurteilung der erstellten Filme erläutert. Darauf aufbauend wird die Gruppierung der Patienten zur Zusammenfassung der Schleimhautbefunde anhand der Intubationsdauer und Ausdehnung der Läsionen vorgestellt. Einen weiteren Bestandteil der Befunderfassung stellt die Nachuntersuchung der Patienten dar.

\subsection{Patientengut}

Der Erfassungszeitraum dieser prospektiven Studie erstreckte sich vom 25. Mai 2007 bis zum 30. Juni 2008. In diesem Zeitraum wurden die Patienten der vorliegenden Analyse auf den anästhesiologisch geführten Intensivstationen 0113 und 0118 des Klinikums Göttingen untersucht. In die Studie aufgenommen wurden Patienten mit einer Mindestintubationsdauer von 24 Stunden, bei denen in den ersten 24 Stunden nach der Extubation eine flexible Tracheo-Laryngoskopie durchgeführt werden konnte.

Als Intubationsdauer wurde ausschließlich der Zeitraum zwischen der jüngsten Intubation und der Extubation erfasst. Bei reintubierten Patienten wurde die Dauer vorheriger Intubationsphasen nicht berücksichtigt. Insgesamt wurden die Aufnahmen von 31 Patienten ausgewertet.

\subsection{Untersuchungsablauf}

Zunächst wurde die Zustimmung der Ethikkommission der Universität Göttingen, Vorsitz Prof. Dr. med. J. Brockmöller, zu diesem Studienvorhaben eingeholt (Reg.Nr.4/11/06).

Die Daten der Patienten, bei denen die Extubation nachts erfolgte oder im Verlauf des Tages vorgesehen war, wurden täglich erfasst. Falls die Patienten nicht verlegt oder wieder reintubiert wurden, untersuchte ein Hals-Nasen-Ohren-Facharzt diese Patienten in den ersten 24 Stunden nach der Extubation auf den Intensivstationen. 
Die Tracheo-Laryngoskopie erfolgte mit einem flexiblen Endoskop 11101 RPC (Karl Storz GmbH \& Co. KG, Tuttlingen). Die Untersuchungen wurden mit dem Visualisierungssystem Atmos CAM 31 (Streute Medizintechnik, Löhne) aufgezeichnet und digitalisiert. Über das Bearbeitungsprogramm rp Szene, Version 6.1n 1999, 2000 (Rheder Software GmbH, Rheder und Partner GmbH Hamburg) erfolgte die Speicherung der Daten auf der Festplatte eines separaten, nur für diese Studie genutzten Computers, Fujitsu Siemens AMILO Pro (Fujitsu Technology Solutions GmbH Headquarters Tokyo).

\subsection{Befunderfassung}

Zum Zwecke der Befunderfassung wurden zwei Dokumentationsbögen entwickelt (s. Abbildungen 3 und 4). Im Rahmen der Studie wurden die aufgezeichneten endoskopischen Untersuchungen anhand der Dokumentationsbögen ausgewertet. Die Ergebnisse aus dieser Auswertung sind Bestandteil des Kapitels 3.

In den folgenden Kapitelabschnitten werden die erfassten Patientendaten sowie das Befundungsprinzip der Laryngoskopie und Tracheoskopie anhand der Dokumentationsbögen erläutert.

\subsubsection{Patientendaten}

Auf dem standardisierten Bewertungsbogen wurden folgende Daten erfasst:

- Name, Alter, Patientennummer

Diese Daten wurden im Zuge der weiteren Auswertung mittels Patientenkennnummern anonymisiert.

\section{- Grunderkrankung}

Als Grunderkrankung wurde die Diagnose mit der dazugehörigen Therapie erfasst, wie beispielsweise einer Operation, die ursächlich für den Aufenthalt auf der Intensivstation war. 


\section{- Intubation}

Ermittelt wurden Datum, Uhrzeit, Grund und Ort der Intubation. Unterschieden wurde zwischen Operationssaal, Intensivstation, Notarztwagen sowie Sonstigen. Intubationsschwierigkeiten und Anzahl der Intubationsversuche wurden ebenfalls festgehalten. Die Anästhesisten wurden gebeten, nach Möglichkeit die Sicht auf die Strukturen des Kehlkopfes bei der Intubation anhand der vier Grade des Cormack-Lehane-Scores zu dokumentieren. Dabei wird unterschieden:

Grad I: $\quad$ Larynxeingang vollständig sichtbar

Grad II: $\quad$ nur hinterer Anteil des Larynxeingangs sichtbar

Grad III: nur Epiglottis sichtbar

Grad IV: $\quad$ nur weicher Gaumen sichtbar

\section{- Extubation}

Dabei wurden ebenfalls Datum, Uhrzeit und Ort der Extubation dokumentiert. Ebenso wurde zwischen Operationssaal, Intensivstation, Notarztwagen und sonstigem Ort unterschieden.

- Tubus

Die verwendete Tubusgröße sowie Firma und Modell des Tubus wurden festgehalten.

\section{- Respiration nach Extubation}

Dokumentiert wurden:

○ 0 eine spontane Normalatmung

○ 1 spontane Dyspnoe

○ 2 durch CPAP assistierte Atmung

○ 3 Dyspnoe trotz Atemunterstützung

○ 4 Re-Intubation

○ $\mathrm{N}$ nicht erfasst 


\subsubsection{Laryngoskopiebefunde}

Die aufgezeichneten Untersuchungen wurden nach dem folgenden Prinzip befundet (s. Abbildungen 3 und 4):

Flexible oder starre $\left(90^{\circ}\right)$ Laryngoskopie

- Diagnose

Läsionen auf Glottisebene wurden beziffert und erfasst:

$\bigcirc \quad$ unauffällige Befunde

○ 1 Veränderungen durch Traumata

Diese Gruppe beinhaltete

$\begin{array}{ccll}\circ & 1.1 & \text { Rötung } \\ \circ & 1.2 & \text { Ulcus } \\ \circ & 1.3 & \text { Hämatom } \\ \circ & 1.4 & \text { Nekrose } \\ \circ & 1.5 & \text { Schleimhautläsionen }\end{array}$

Ebenfalls dokumentiert wurden:

$\circ 2$ Granulation

○ 3 Synechie

○ 4 Ödeme

$\circ 5 \quad$ Fibrin

○ 6 Malazie

○ 7 Stenose

Nicht beurteilbare Aufnahmen erhielten die Bezeichnung (N).

- Lokalisation

Die anatomischen Strukturen des Kehlkopfes wurden nummeriert. Somit konnten die Diagnosecodes den genauen Lokalisationscodes zugeordnet werden.

\section{- Glottiseinblick und Verschleimung}

Festgehalten wurde die Einsehbarkeit der glottischen Strukturen. Eine verminderte Einsehbarkeit wurde je nach Ausmaß der Einschränkung aufsteigend nummeriert.

Der Verschleimungsgrad wurde ebenfalls dokumentiert. 


\section{- Stimmlippenmobilität}

Auf diesem Parameter lag das Hauptaugenmerk der Studie. Ab- und Adduktionsstörungen der Stimmlippen sowie paramediane und intermediäre Stillstände wurden dokumentiert.

\section{- Phonationsebene und Taschenfaltenaktivität}

Folgende Phonationsebenen wurden unterschieden:
$\circ \quad 0 \quad$ glottisch, Normalbefund
○ 1 ventrikulär
○ $\quad 1.2$ Zwischenstufe ventrikulo-supraventrikulär
○ 2 supraventrikulär
○ 10 glottoventrikulär
○ 20 glotto-supraventrikulär
○ N nicht beurteilbar

Die Taschenfaltenaktivität wurde ebenfalls beurteilt.

\section{- Stimme}

Die Eigenschaften des Stimmklangs, Rauigkeit, Behauchtheit und Heiserkeit wurden ebenfalls begutachtet. Die Beurteilung erfolgte anhand einer fünfstufigen Skala (nach Seidner und Eysholdt 2005):
○ $0 \quad$ keine Störung
○ 1 geringe Störung
○ 2 mittelgradige Störung
○ 3 starke Störung
○ 4 Aphonie
$\circ \mathrm{N}$ nicht beurteilbar 


\subsubsection{Tracheoskopiebefunde}

Die Befunde der Tracheoskopie wurden vergleichbar denen der Laryngoskopie dokumentiert (s. Abbildungen 3 und 4):

- Flexible oder starre $\left(90^{\circ}\right)$ Endoskopie

- Diagnose

Es wurden die gleichen Diagnosen nach demselben Schema wie bei der Laryngoskopie erfasst (s. Kapitel 2.3.2).

- Lokalisation

Die Trachealspangen wurden durchnummeriert und jeweils zwei einer Kennziffer zugeordnet. Nicht beurteilbare Befunde erhielten die Kennzeichnung (N).

- Trachea-Einblick

Sichteinschränkungen wurden dem Ausmaß entsprechend dokumentiert. Untersuchungen, bei denen die Trachea nicht dargestellt werden konnte, erhielten die Kennzeichnung (N).

\subsubsection{Qualitätsbeurteilung der Aufnahmen}

Die Aufnahmequalität der erstellten Filme und die daraus resultierende Beurteilbarkeit der organischen und funktionellen Befunde wurden auf einer Skala von 1 (schlecht) bis 10 (gut) erfasst (s. Abbildung 4). 


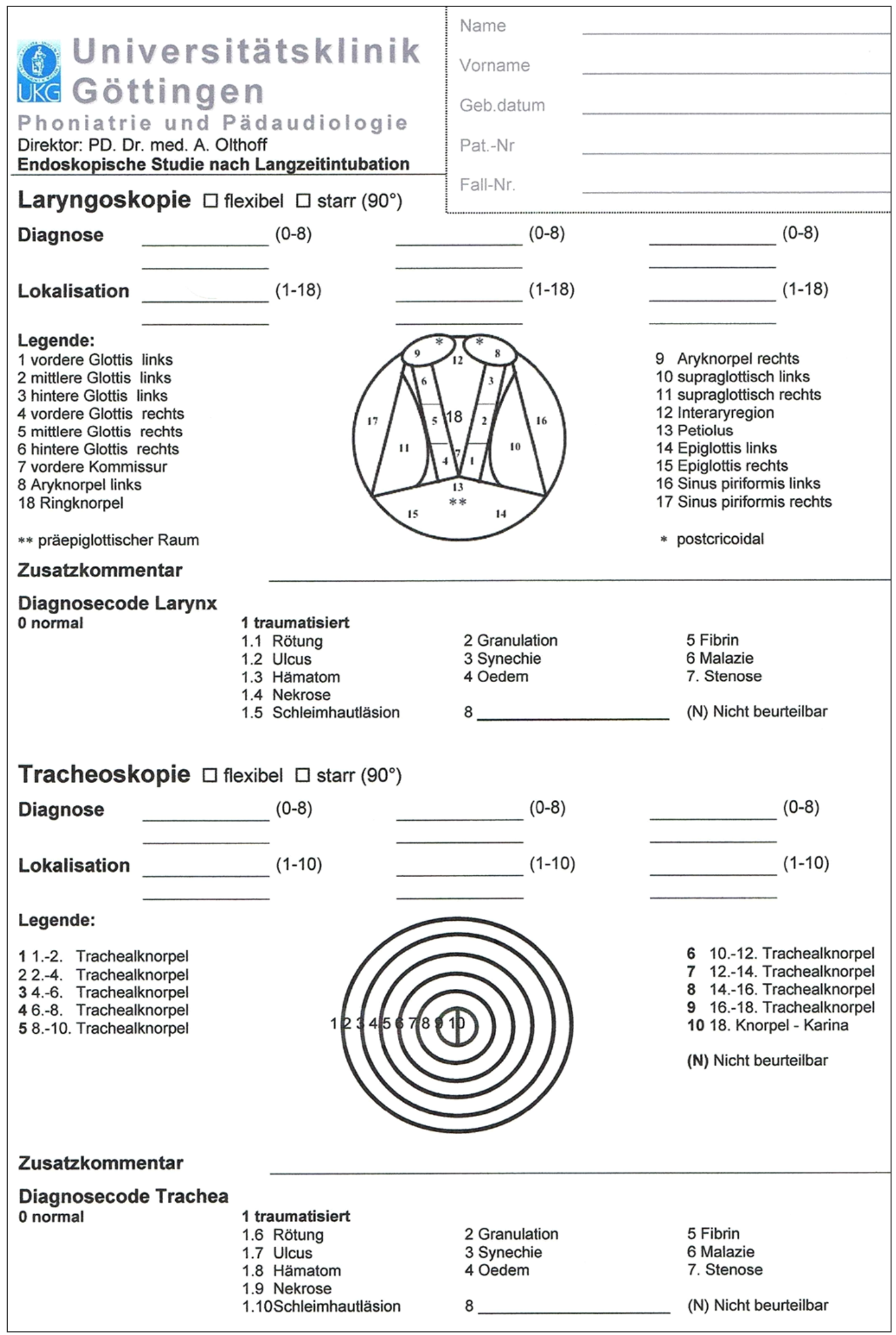

Abbildung 3: Dokumentationsbogen I zur Erfassung der endoskopischen Befunde (Quelle: eigene Entwicklung) 


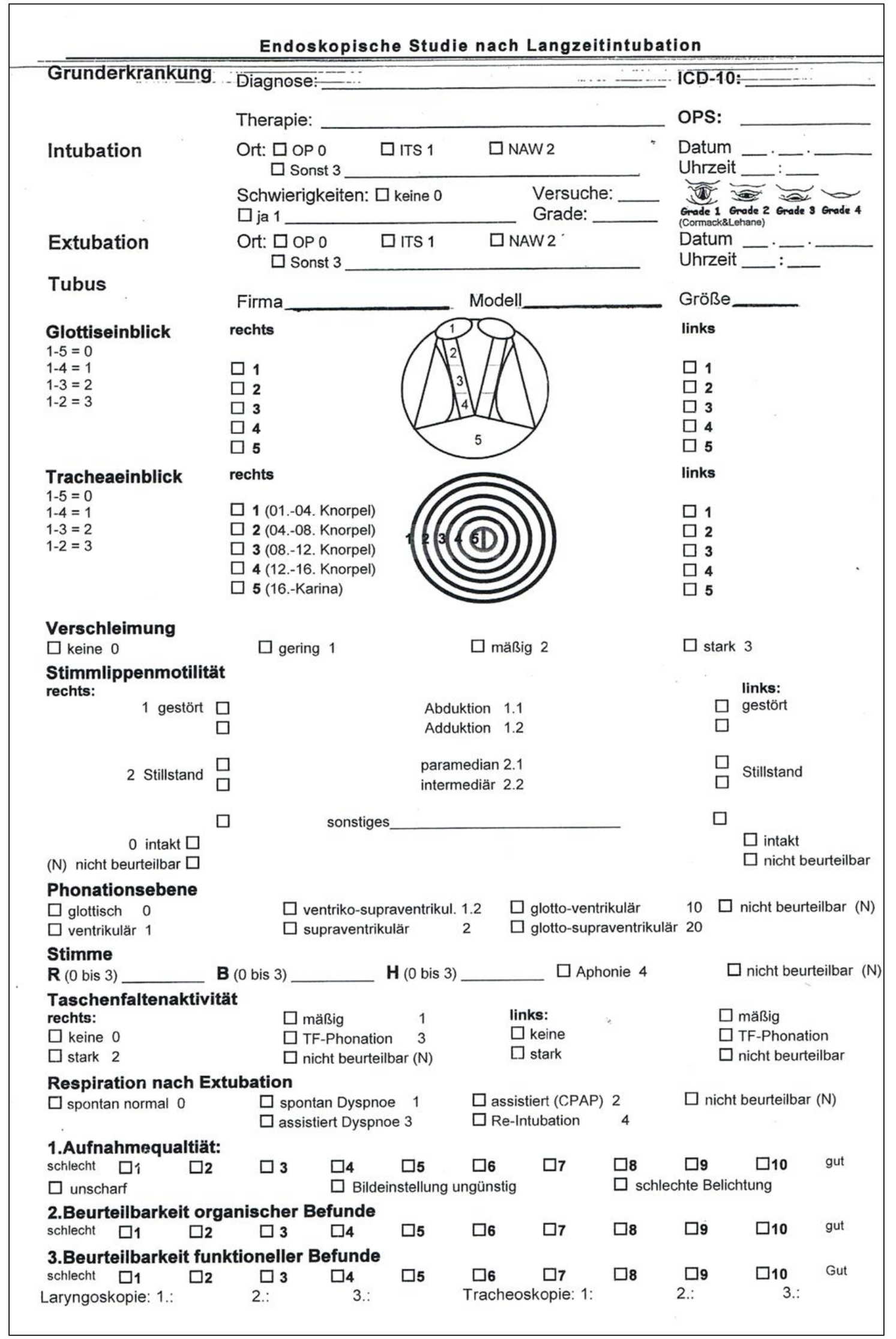

Abbildung 4: Dokumentationsbogen II zur Erfassung der endoskopischen Befunde, Untersuchungsaufnahmequalität, Grunderkrankung der Patienten und Stimmbewertung (Quelle: eigene Entwicklung) 


\subsection{Zusammenfassung der Schleimhautbefunde}

In der Zusammenfassung der Schleimhautbefunde wurden Patienten anhand der Intubationsdauer in Anlehnung an die Untersuchung von Burns et al. (1979) in vier Gruppen eingeteilt.

Gruppe I: $\quad 24-47 \mathrm{~h}$

Gruppe II: $\quad 48-71 \mathrm{~h}$

Gruppe III: $\quad 72-120 \mathrm{~h}$

Gruppe IV: $\quad>120 \mathrm{~h}$

Zusätzlich erfolgte eine Zuordnung der Schleimhautbefunde nach Ausdehnung und Tiefe der Verletzung zu drei Schweregraden in Anlehnung an die Auswertungsskala von Lindholm (1969).

Grad I: Verletzungen mit intakter Mukosa: Ödeme, Rötung

Grad II: Mukosa durchdringende Verletzungen $(<1 / 3$ des Atemwegsumfangs)

Grad III: Mukosa durchdringende Verletzungen (> 1/3 des Atemwegsumfangs)

\subsection{Nachuntersuchung}

Im Februar 2010 wurden die Patienten, bei denen eine Bewegungsstörung der Stimmlippen festgestellt worden war, zur Nachuntersuchung in die HNO-Klinik, Abteilung Phoniatrie und Pädaudiologie der Universität Göttingen gebeten. Die Nachuntersuchung war zur Verlaufsbeurteilung beobachteter Bewegungsstörungen vorgesehen. 


\section{Ergebnisse}

Im folgenden Kapitel werden die anhand der beschriebenen Methodik erhobenen Befunde vorgestellt. Zunächst erfolgt die Beschreibung des Patientenkollektivs. Im Weiteren werden zum einen die laryngoskopischen Befunde der Schleimhaut, unterteilt in Läsionen, deren Lokalisationen und Ausdehnung, und zum anderen die Bewegungsstörungen der Stimmlippen jeweils mit Dokumentation der Intubationsdauer beschrieben. Ebenso erfolgt die Darstellung der Tracheoskopiebefunde. Die Qualität der Aufnahmen ist bereits teilweise in den oben genannten Abschnitten berücksichtigt. Eine zusammenfassende Darstellung ist ebenfalls aufgeführt.

Abschließend werden die Ergebnisse der Nachuntersuchungen vorgestellt.

\subsection{Patientenkollektiv}

Insgesamt wurden 31 Patienten endoskopiert, darunter waren 23 Männer und 8 Frauen. Das Durchschnittsalter des Patientenkollektivs betrug 70,7 Jahre (55 bis 83 Jahre). 26 der untersuchten Patienten waren zur postoperativen Überwachung auf die Intensivstation verlegt worden. 19 dieser Patienten hatten sich im Vorfeld endothorakalen Eingriffen im Rahmen von Herzklappen- und Bypassoperationen unterzogen. 16 dieser 26 Patienten blieben postoperativ intubiert, 9 mussten bei insuffizienter Respiration im Verlauf reintubiert werden. Ein Patient wurde postoperativ intubiert aus einer anderen Klinik übernommen.

Bei den übrigen 5 der 31 Patienten führten schwere systemische Erkrankungen oder intrakranielle Blutungen zur Verlegung auf die Intensivstation und dort zur Intubation oder Re-Intubation. Auch hier war in einem Fall einen Monat zuvor ein endothorakaler Eingriff erfolgt.

Die mittlere Intubationsdauer betrug 80,8 \pm 68,57 Stunden (24 bis 255 Stunden). Bei keinem Patienten wurden von den Anästhesisten Intubationsschwierigkeiten angegeben. In 30 Fällen wurde ein Portex® PVC Tubus (Smith-Medical, St. Paul USA) verwandt, ein Patient erhielt einen Woodbrige-Tubus (Covidien Neustadt a. d. Donau). Bei 14 Patienten war die Respiration nach Extubation spontan und normal, ein Patient entwickelte eine leichte Dyspnoe. Weitere 14 Patienten benötigten eine CPAP-Beatmung. Zwei Patienten mussten reintubiert werden. 
In Tabelle 3 werden die Patientendaten nach Intubationsindikation gruppiert zusammenfassend dargestellt.

Tabelle 3: $\quad$ Daten des Patientenkollektivs $(n=31)$

Zeichenerklärung: Nr.: Patientenkennnummer, gleichbleibend in der ganzen Arbeit; a: Jahre; CPAP: Continuous Positive Airway Pressure; h: Stunde; m: männlich; OP: Operation; P: Portex-Doppellumen-Tubus; w: weiblich; WB: Woodbrige-Spiral-Tubus; * Zustand nach einem endothorakalen Eingriff

\begin{tabular}{|c|c|c|c|c|c|c|}
\hline Nr. & $\begin{array}{l}\text { Intubations- } \\
\text { indikation }\end{array}$ & Geschlecht & Alter (a) & $\begin{array}{l}\text { Intubations- } \\
\text { dauer(h) }\end{array}$ & Tubus & $\begin{array}{l}\text { Respiration } \\
\text { nach Extubation }\end{array}$ \\
\hline 1 & OP* & $\mathrm{m}$ & 69 & 24 & P & spontan, normal \\
\hline 2 & OP* & $\mathrm{m}$ & 78 & 26 & P & spontan, normal \\
\hline 3 & OP* & $\mathrm{m}$ & 73 & 26 & P & CPAP \\
\hline 4 & OP* & $\mathrm{m}$ & 76 & 26 & P & CPAP \\
\hline 5 & OP* & $\mathrm{m}$ & 83 & 28 & P & spontan, normal \\
\hline 6 & OP* & $\mathrm{m}$ & 79 & 30 & P & spontan, normal \\
\hline 7 & OP* & $\mathrm{m}$ & 79 & 30 & $P$ & spontan, normal \\
\hline 8 & $\mathrm{OP}^{*}$ & $\mathrm{~m}$ & 56 & 34 & P & spontan, normal \\
\hline 9 & OP* & $\mathrm{m}$ & 76 & 48 & $P$ & spontan, normal \\
\hline 10 & OP* & $\mathrm{m}$ & 69 & 54 & P & spontan, normal \\
\hline 11 & OP & $\mathrm{m}$ & 80 & 58 & P & CPAP \\
\hline 12 & OP & $\mathrm{m}$ & 70 & 57 & $P$ & CPAP \\
\hline 13 & OP & w & 82 & 72 & WB & spontan, normal \\
\hline 14 & OP* & $\mathrm{m}$ & 76 & 139 & $P$ & Re-Intubation \\
\hline
\end{tabular}




\begin{tabular}{|c|c|c|c|c|c|c|}
\hline Nr. & $\begin{array}{l}\text { Intubations- } \\
\text { indikation }\end{array}$ & Geschlecht & Alter (a) & $\begin{array}{l}\text { Intubations- } \\
\text { dauer(h) }\end{array}$ & Tubus & $\begin{array}{l}\text { Respiration } \\
\text { nach Extubation }\end{array}$ \\
\hline 15 & OP* & w & 64 & 213 & $P$ & Re-Intubation \\
\hline 16 & OP & $\mathrm{m}$ & 83 & 236 & $P$ & spontan, normal \\
\hline 17 & Re-Intubation* & $\mathrm{m}$ & 63 & 35 & $P$ & CPAP \\
\hline 18 & Re-Intubation & w & 68 & 39 & $P$ & CPAP \\
\hline 19 & Re-Intubation* & $\mathrm{m}$ & 77 & 39 & $P$ & CPAP \\
\hline 20 & Re-Intubation* & $\mathrm{m}$ & 76 & 48 & $P$ & spontan, normal \\
\hline 21 & Re-Intubation & $\mathrm{m}$ & 60 & 73 & $P$ & spontan, normal \\
\hline 22 & Re-Intubation* & $\mathrm{m}$ & 55 & 128 & $P$ & CPAP \\
\hline 23 & Re-Intubation* & w & 69 & 132 & $P$ & CPAP \\
\hline 24 & Re-Intubation & w & 62 & 223 & $P$ & spontan, Dyspnoe \\
\hline 25 & Re-Intubation* & w & 57 & 255 & $P$ & CPAP \\
\hline 26 & $\begin{array}{l}\text { Intubiert } \\
\text { aufgenommen* }\end{array}$ & $\mathrm{m}$ & 75 & 79 & $P$ & CPAP \\
\hline 27 & Resp. Insuff* & w & 67 & 38 & $P$ & CPAP \\
\hline 28 & Resp. Insuff & w & 60 & 48 & $P$ & spontan, normal \\
\hline 29 & $\begin{array}{l}\text { Resp. Insuff } \\
\text { (Re-Intubation) }\end{array}$ & $\mathrm{m}$ & 63 & 52 & $P$ & CPAP \\
\hline 30 & Resp. Insuff. & $\mathrm{m}$ & 65 & 66 & $P$ & CPAP \\
\hline 31 & $\begin{array}{l}\text { Resp. Insuff } \\
\text { (Re-Intubation) }\end{array}$ & $\mathrm{m}$ & 82 & 149 & $P$ & spontan, normal \\
\hline
\end{tabular}




\subsection{Laryngoskopische Befunde}

\subsubsection{Schleimhautläsionen}

Sämtliche 31 Patienten wurden flexibel endoskopiert. Bei 29 der untersuchten Patienten wurden Schleimhauttraumata im Glottisbereich festgestellt. In zwei Fällen war die Beurteilung der Glottisschleimhaut in der Laryngoskopieaufnahme nicht möglich. Eine Verletzung der Schleimhäute war damit bei diesen Patienten nicht auszuschließen.

21 der 29 Patienten hatten ein Ödem im Larynxbereich. Bei 16 Patienten fanden sich Rötungen. Ebenso wiesen 16 Patienten Fibrin auf. Schleimhautläsionen zeigten sich bei fünf Patienten.

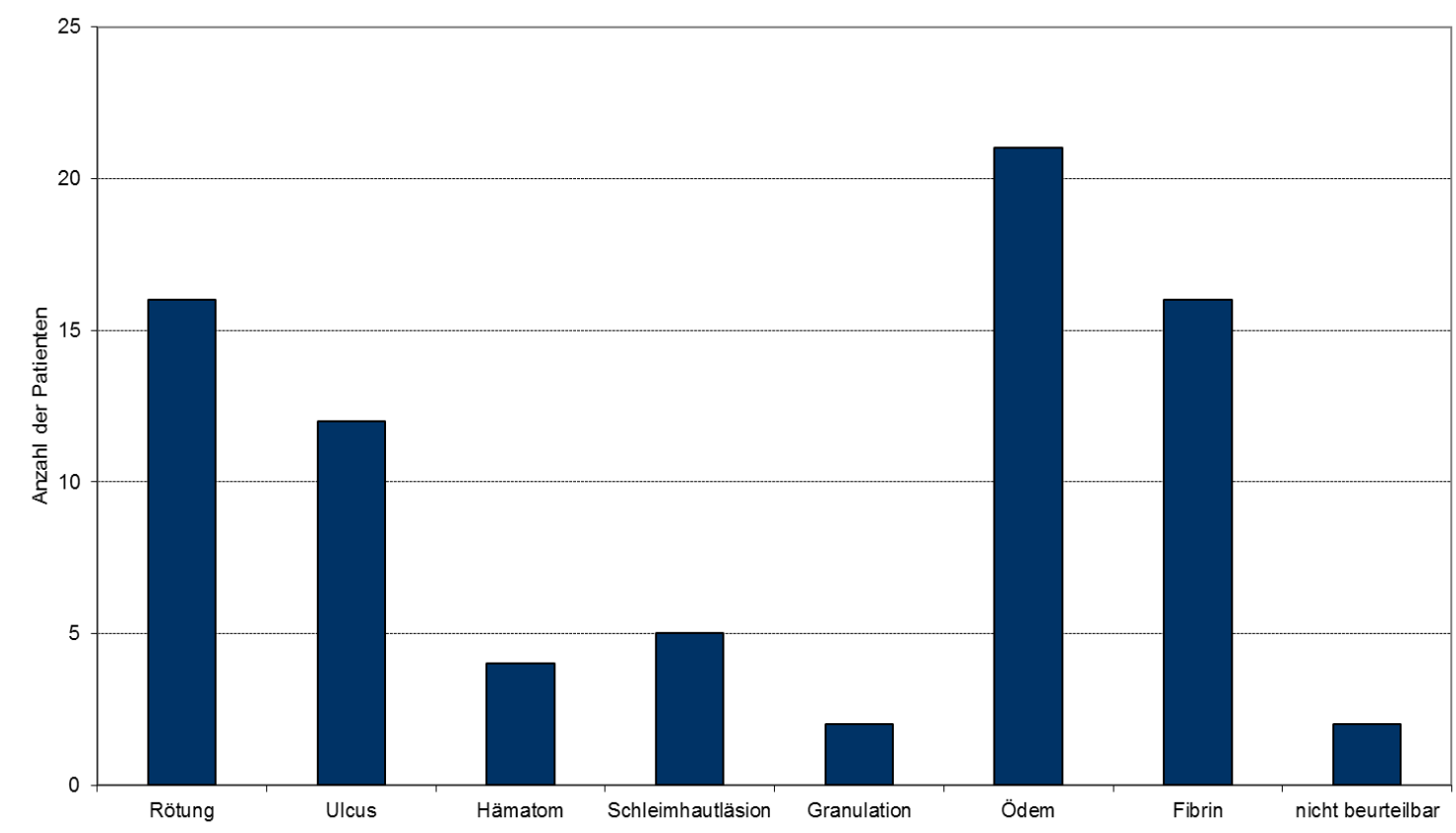

Abbildung 5: Schleimhautbefunde der Laryngoskopie $(n=31)$

Ödeme traten gehäuft im Bereich der Aryknorpel und der Interaryregion, der Glottis, vereinzelt auch in der supraglottischen Region auf. Im Bereich der hinteren Glottis wurden bei vielen Patienten Ulzerationen, teilweise mit Fibrinauflagerungen, Rötungen und Schleimhautläsionen festgestellt. Vereinzelt zeigten sich auch Rötungen, Ulzerationen und Fibrinbeläge beidseitig in der Aryregion und subglottisch im Bereich des Ringknorpels. Bei vier Patienten fanden sich Glottishämatome. Zwei dieser Hämatome waren rechtsseitig, eins linksseitig. In einem Fall fand sich beidseitig ein Hämatom.

In zwei Fällen traten im Bereich der hinteren Glottis Granulationen auf. Bei nasotracheal intubierten Patienten wurden außerdem schwere Läsionen der Nasenschleimhaut 
festgestellt. In einem Fall lag bei komplett atrophierter Schleimhaut der Septumknorpel einseitig frei. Die Schleimhauttraumata sind in den Abbildungen 5 und 6 zusammenfassend dargestellt.
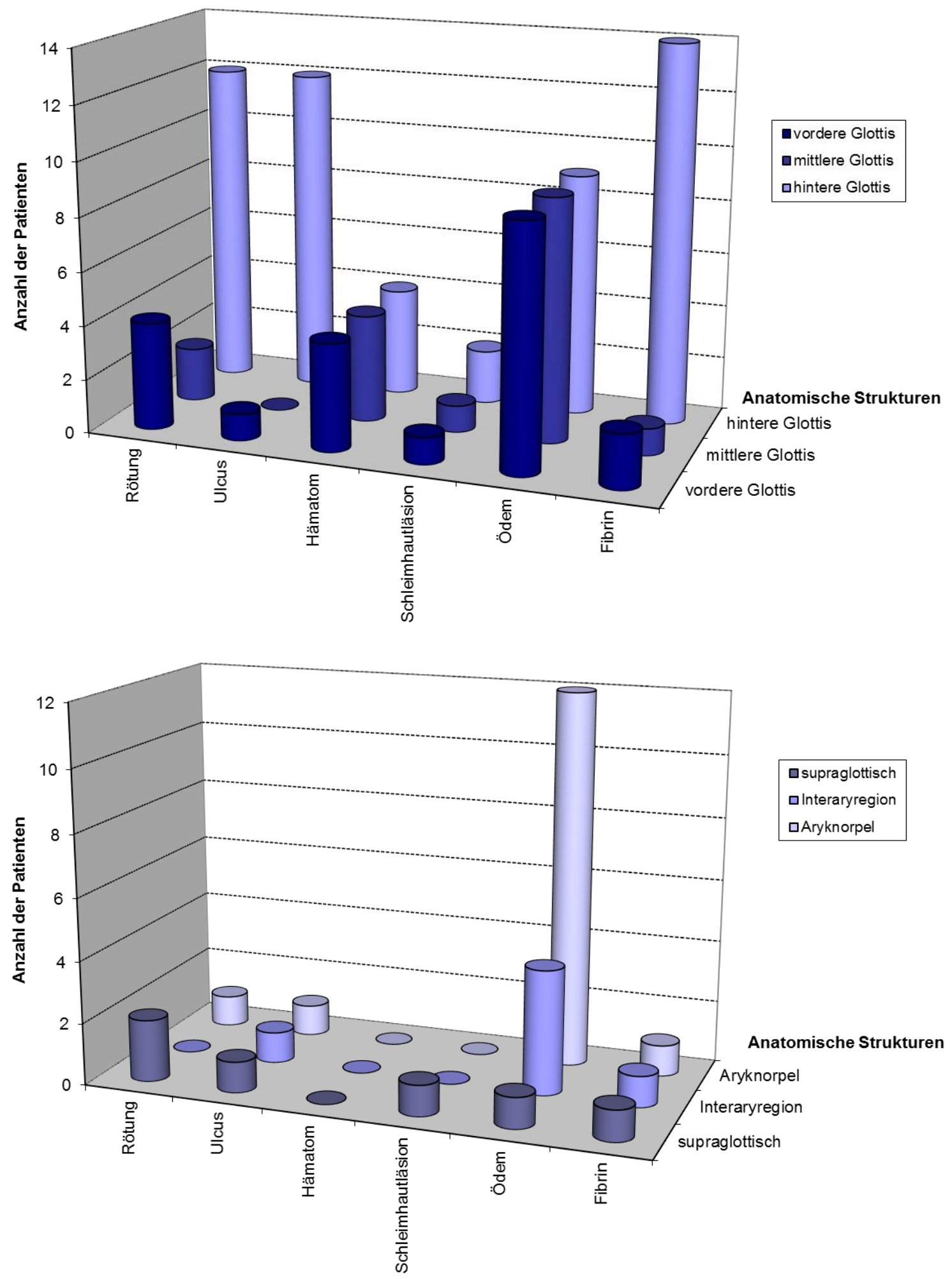

Abbildung 6: Schleimhautbefunde der Laryngoskopie, nach Lokalisation geordnet $(n=31)$ 
Die folgende Tabelle 4 zeigt eine Zusammenfassung der Befunde im Hinblick auf Tiefe und Ausdehnung der Verletzung in Anlehnung an die Befundauswertung von Lindholm (1969), betrachtet im Zusammenhang mit der Intubationsdauer. Die Gruppierung des Patientenkollektivs nach Intubationsdauer erfolgte anhand der Einteilung von Burns et al. (1979). Ergänzend werden in Abbildung 7 beispielhafte Aufnahmen von Schleimhautläsionen des Larynx unter Berücksichtigung der Intubationsdauer gezeigt.

Tabelle 4: Zusammenfassung der Schleimhautbefunde unter Berücksichtigung von Intubationsdauer (h) und Verletzungsausdehnung

\begin{tabular}{|c|c|c|c|c|}
\hline & $\begin{array}{l}\text { Gruppe I } \\
(24-47 \text { h) }\end{array}$ & $\begin{array}{l}\text { Gruppe II } \\
(48-71 \text { h) }\end{array}$ & $\begin{array}{l}\text { Gruppe III } \\
(72-120 \text { h) }\end{array}$ & $\begin{array}{l}\text { Gruppe IV } \\
(>120 \mathrm{~h})\end{array}$ \\
\hline $\begin{array}{l}\text { Grad I } \\
\text { Verletzungen mit intakter } \\
\text { Mukosa: Ödeme, Rötung }\end{array}$ & 4 & 4 & - & 1 \\
\hline $\begin{array}{l}\text { Grad II } \\
\text { Mukosa durchdringende } \\
\text { Verletzung: } \\
\text { ( }<1 / 3 \text { des Atemwegumfangs) }\end{array}$ & 5 & 4 & - & 5 \\
\hline $\begin{array}{l}\text { Grad III } \\
\text { Mukosa durchdringende } \\
\text { Verletzung: } \\
\text { ( }>1 / 3 \text { des Atemwegumfangs) }\end{array}$ & 1 & - & 3 & 2 \\
\hline Nicht beurteilbar & 2 & - & - & - \\
\hline
\end{tabular}

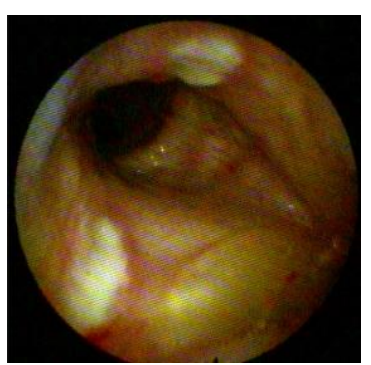

A

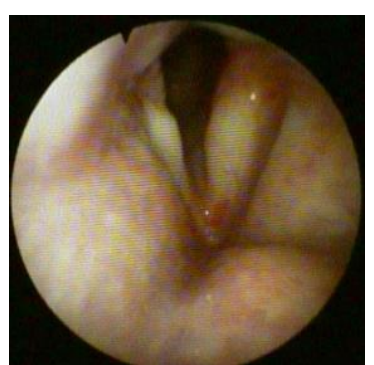

B

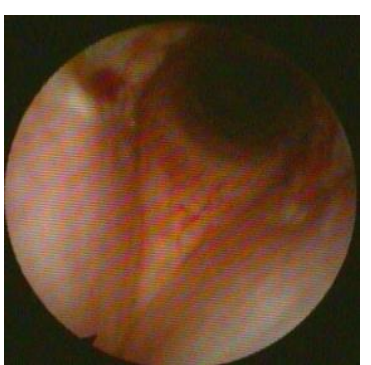

C

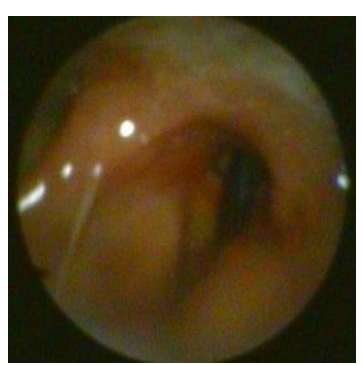

D

Abbildung 7: Schleimhautläsionen des Larynx
A: $30 \mathrm{~h}$ Intubationsdauer (Pat.Nr. 7)
B: $30 \mathrm{~h}$ Intubation (Pat.Nr. 6)
C: 223 h Intubationsdauer (Pat.Nr. 24)
D: $48 \mathrm{~h}$ Intubationsdauer (Pat.Nr. 20) 


\subsubsection{Bewegungsstörungen der Stimmlippen}

Unter Bewegungsstörungen wurden Ab- und Adduktionsstörungen sowie Stillstände der Stimmlippen zusammengefasst. Bei 14 der 31 untersuchten Patienten wurden Bewegungsstörungen der Stimmlippen festgestellt. In fünf Fällen waren diese Bewegungsstörungen beidseitig, bei neun Patienten einseitig. Eine Bewegungsstörung der linken Stimmlippe zeigten sieben dieser neun Patienten. Die Beweglichkeit der rechten Stimmlippe war in zwei Fällen eingeschränkt. Bei neun der Patienten, die Mobilitätsstörungen der Stimmlippen aufwiesen, war zuvor ein endothorakaler Eingriff erfolgt. In Tabelle 5 sind die genauen Diagnosen, die erfolgten Eingriffe und Behandlungen sowie die Intubationsdauer der Patienten mit Mobilitätstörungen dargestellt. Die unterschiedlichen Formen der Bewegungsstörungen sind ebenfalls aufgeführt. 
Tabelle 5: Bewegungsstörungen der Stimmlippen

Zeichenerklärung: ACVB: Aortokoronarer Venenbypass; Ak: Aortenklappe;

AKE: Aortenklappenersatz; KHK: Koronare Herzkrankheit; Li: Links;

MI: Mitralklappeninsuffizienz; MKE: Mitralklappenersatz; Nr.: Patientenkennung;

(p): paramedian; pAVK: periarterielle Verrschlusskrankheit; Re: Rechts;

Z.n. Zustand nach

\begin{tabular}{|c|c|c|c|c|}
\hline Nr. & $\begin{array}{l}\text { Stimm- } \\
\text { lippen- } \\
\text { mobilität }\end{array}$ & $\begin{array}{l}\text { Intubations- } \\
\text { dauer (h) }\end{array}$ & Diagnose & $\begin{array}{l}\text { Gestörter } \\
\text { Bewegungs- } \\
\text { ablauf }\end{array}$ \\
\hline 5 & Beidseits & 28 & $\begin{array}{l}\text { Z.n. endothorakalem Eingriff } \\
\text { (ACVB 3x) bei KHK }\end{array}$ & $\begin{array}{l}\text { Li: Abduktion. } \\
\text { Re: Abduktion }\end{array}$ \\
\hline 17 & Beidseits & 35 & $\begin{array}{l}\text { Z.n. endothorakalem Eingriff } \\
\text { (Aortenbogenersatz) bei Dissektion }\end{array}$ & $\begin{array}{l}\text { Li: Stillstand }(p) \\
\text { Re: Abduktion }\end{array}$ \\
\hline 20 & Beidseits & 48 & $\begin{array}{l}\text { Z.n. endothorakalem Eingriff } \\
\text { (ACVB 3x) bei KHK }\end{array}$ & $\begin{array}{l}\text { Li: Stillstand }(p) \\
\text { Re: Abduktion }\end{array}$ \\
\hline 11 & Beidseits & 58 & $\begin{array}{l}\text { Z.n. Y-Prothese bei } \\
\text { Bauchaortenaneurysma }\end{array}$ & $\begin{array}{l}\text { Li: Stillstand }(p) \\
\text { Re: Stillstand }(p)\end{array}$ \\
\hline 14 & Beidseits & 139 & $\begin{array}{l}\text { Z.n. endothorakalem Eingriff } \\
\text { (MKE, ACVB } 3 x \text { ) bei MI }\end{array}$ & $\begin{array}{l}\text { Li: Abduktion } \\
\text { Re: Abduktion }\end{array}$ \\
\hline 2 & Links & 26 & $\begin{array}{l}\text { Z.n. endothorakalem Eingriff } \\
\text { (AKE) bei AK-Stenose }\end{array}$ & Abduktion \\
\hline 19 & Links & 39 & $\begin{array}{l}\text { Z.n. endothorakalem Eingriff } \\
\text { (AKE, ACVB 2x) bei AK-Stenose, } \\
\text { KHK }\end{array}$ & Abduktion \\
\hline 29 & Links & 52 & $\begin{array}{l}\text { Z.n. Krampfanfall } \\
\text { bei intrakranieller Blutung }\end{array}$ & Stillstand (p) \\
\hline 12 & Links & 57 & $\begin{array}{l}\text { Z.n. Laparotomie } \\
\text { bei Zwerchfellhernie }\end{array}$ & Adduktion \\
\hline 21 & Links & 73 & $\begin{array}{l}\text { Z.n. Art. Femoralis-profunda-plastik } \\
\text { bei pAVK }\end{array}$ & Stillstand $(p)$ \\
\hline 22 & Links & 128 & $\begin{array}{l}\text { Z.n. endothorakalem Eingriff } \\
\text { (AKE, ACVB 2x) bei AK-Stenose, } \\
\text { KHK }\end{array}$ & Adduktion \\
\hline 15 & Links & 213 & $\begin{array}{l}\text { Z.n. endothorakalem Eingriff } \\
\text { (MKE) bei komb. } \\
\text { Mitralklappenvitium }\end{array}$ & Abduktion \\
\hline 27 & Rechts & 38 & $\begin{array}{l}\text { Z.n. endothorakalem Eingriff } \\
\text { V.a. Pneumothorax rechts }\end{array}$ & Abduktion \\
\hline 28 & Rechts & 48 & Z.n. Hirnstammblutung & Stillstand (p) \\
\hline
\end{tabular}




\subsubsection{Stimmqualität}

Eine suffiziente Tonerfassung und somit Beurteilbarkeit der Stimmqualität gelang in den Endoskopien nicht. Das Hauptaugenmerk wurde auf die Zielsetzung der Untersuchung und somit auf die Auswertung der laryngoskopischen Befunde gelegt.

\subsection{Befunde der Tracheoskopie}

13 der 31 Patienten konnten flexibel tracheoskopiert werden. Bei den übrigen Patienten waren die Schutzreflexe zu stark ausgeprägt, so dass keine verwertbaren Aufnahmen erstellt werden konnten. Bei fünf Patienten war die Trachealschleimhaut ohne Verletzungen. Bei den übrigen acht Patienten fanden sich Rötungen, Ulzerationen, teilweise mit Fibrinbelägen und Schleimhautläsionen. Eine Übersicht der Schleimhautbefunde der Tracheoskopie ist in Abbildung 8 gegeben.

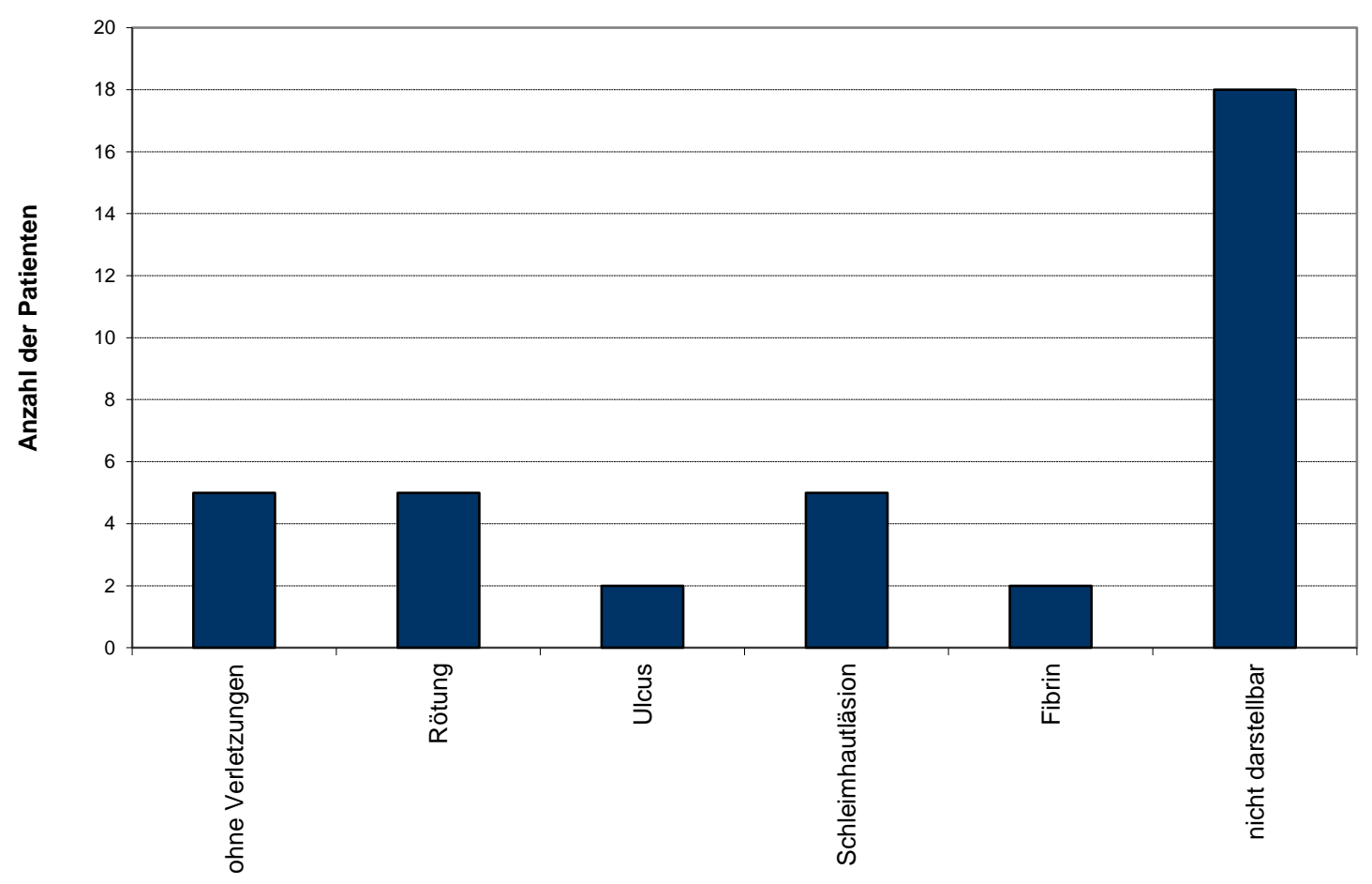

Abbildung 8: Schleimhautbefunde der Tracheoskopie $(n=31)$ 
Ein beispielhafter Befund aus einer Tracheoskopie ist in Abbildung 9 dargestellt. Die Abbildung zeigt eine Trachealläsion des Patienten Nr. 19 nach 39 Intubationsstunden mit Rötung und Schleimhautläsion im Bereich des oberen Trachealknorpels. Typischerweise werden derartige Verletzungen durch den Tubuscuff verursacht.

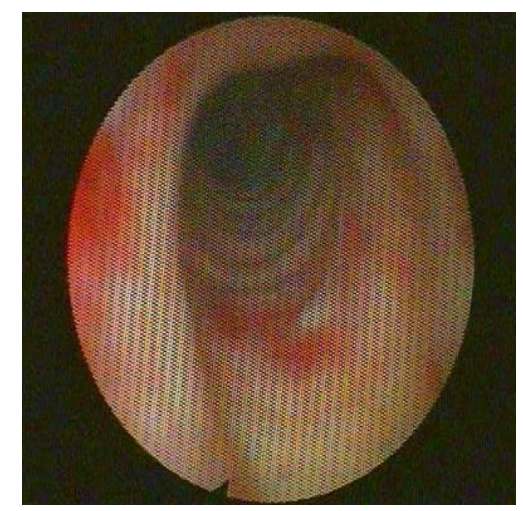

Abbildung 9: Trachealläsion nach $39 \mathrm{~h}$ Intubation (Pat.Nr. 19)

\subsection{Qualitätsbeurteilung der Aufnahmen}

Die Aufnahmequalität der Laryngoskopien war durchschnittlich gut, der Mittelwert lag bei 8. In zwei Fällen war die Beurteilung wegen ungünstiger Bildeinstellung und mangelnder Schärfe erschwert. Die Beurteilbarkeit der organischen und funktionellen Befunde war mit einem Durchschnittswert von 8 ebenfalls gut. Die Aufnahmequalität und Beurteilbarkeit der organischen Befunde bei den 13 Tracheoskopien lag im Mittel bei 7. Aufgrund schlechter Belichtung war die Beurteilung bei vier der 13 Patienten erschwert. Insgesamt konnten die Trachea nur unzureichend häufig und genau dargestellt werden. Auch die Darstellungstiefe in der Trachea war bei sämtlichen Aufnahmen unterschiedlich.

\subsection{Nachuntersuchung}

Von den 14 Patienten mit Bewegungsstörungen waren drei zum geplanten Zeitpunkt der Nachuntersuchung bereits verstorben. Die übrigen Patienten wurden nach Rücksprache mit dem jeweiligen Hausarzt und telefonischer Kontaktaufnahme schriftlich zu einer Nachuntersuchung gebeten. Einige Patienten konnten aufgrund eines schlechten Allgemeinzustandes oder wegen der Entfernung des Wohnortes nicht an der Nachuntersuchung teilnehmen. Ein Großteil konnte nicht kontaktiert werden und meldete 
sich auch nicht auf die schriftliche Anfrage. Nur ein Patient (Pat. Nr. 17) mit initial beidseitiger Mobilitätsstörung, mit linksseitiger Stimmbandlähmung und rechtsseitiger Abduktionsstörung erschien zur Nachuntersuchung. Hier zeigte sich ein Jahr nach Extubation ein Normalbefund. 


\section{Diskussion}

Die dieser Arbeit zugrunde liegende Studie wurde mit dem Ziel durchgeführt, die Häufigkeit und den Schweregrad auftretender Bewegungsstörungen der Stimmlippen und laryngealer Verletzungen nach Langzeitintubation darzustellen. Im Zuge dieser Arbeit wurde zudem die mögliche Korrelation auftretender laryngealer Komplikationen mit der Intubationsdauer untersucht.

Die Schleimhautläsionen des Larynx, die dokumentierten Bewegungsstörungen sowie die Befunde der Trachea werden in den nachfolgenden Abschnitten unter Berücksichtigung der Ergebnisse aus der Literatur verglichen und diskutiert.

\subsection{Schleimhautläsionen des Larynx}

Schleimhautbefunde des Larynx nach Langzeitintubation sind bereits mehrfach in umfangreichen Studien dokumentiert. Hierbei finden sich unterschiedliche Angaben zu Häufigkeit und Schweregrad der Läsionen besonders in Zusammenhang mit der Intubationsdauer.

Colice et al. (1989) dokumentierten Schleimhautbefunde des Larynx nach einer Mindestintubationsdauer von vier Tagen. $94 \%$ der untersuchten Patienten (77 von 82 Patienten) wiesen Verletzungen auf. Zum Großteil (58\%) bestanden milde Schäden. Unter milden Schäden wurden Veränderungen mit intakter Mukosa sowie oberflächliche Mukosaschäden zusammengefasst. Etwa zu gleichen Teilen fanden sich moderate (29\%) sowie schwerste Schäden (23\%) mit freiliegendem Knorpel.

Lindholm (1969) dokumentierte nach Langzeitintubation (min. 24 h) bei sämtlichen 161 untersuchten Patienten Mukosaverletzungen. Hauptsächlich fanden sich Verletzungen II. Grades (s. Tabelle 1).

In der vorliegenden Untersuchung waren die Schleimhautbefunde von 2 der 31 untersuchten Patienten nicht beurteilbar. Die übrigen 29 Patienten wiesen Schleimhautläsionen auf. Somit ist bereits feststellbar, dass sämtliche Patienten mit beurteilbaren Befunden Läsionen aufgrund der Intubation aufwiesen.

Der Schwere der Verletzung nach aufsteigend geordnet, fanden sich bei 21 Patienten Ödeme, bei 16 Patienten Rötungen, bei 16 Patienten Fibrinauflagerungen sowie bei 12 Patienten Ulzerationen. Fünf Patienten zeigten Schleimhautläsionen. Bei zwei Patienten wurden Granulome dokumentiert. Die Hauptlokalisation der Verletzungen waren die 
hintere Glottis sowie die Aryknorpel und die Ringknorpelebene, wobei schwerere Verletzungen wie Ulzerationen und Fibrinauflagerungen hauptsächlich im Bereich der hinteren Glottis zur Darstellung kamen.

Die hintere Kommissur ist durch die Kehlkopfanatomie bedingte dorsale Tubuslage im Laryx besonders belastet. Eine schmale Auflagefläche und die dortige mögliche Druckbelastung von bis zu $400 \mathrm{mmHg}$, gemessen im Hundeversuch (Weymuller et al. 1983), die den kapillaren Schleimhautperfusionsdruck von ungefähr $30 \mathrm{mmHg}$ (Weymuller et al. 1983) deutlich überschreitet, sind hinreichend erklärend für die dargestellten Befunde der hinteren Glottis, Aryknorpel und Interaryregion.

Die Ringknorpelebene ist eine anatomisch bedingte subglottische Engstelle. Der Tubus muss sich an dieser Stelle der Anatomie entsprechend umformen. Eine Druckbelastung der dortigen Schleimhaut durch den Tubus bis zu 90 mmHg ist von Stenqvist et al. (1979) in einem Hasenversuch nachgewiesen.

Mit den genannten Lokalisationen Glottis, Aryknorpel, Interaryregion und Ringknorpelebene entsprechen auch in der vorliegenden Untersuchung die Hauptlokalisationen der Schleimhautverletzung den Literaturangaben von Benjamin und Holinger (2008) Burns et al. (1979), Colice et al. (1989), Ellis und Bennett (1977) sowie Lindholm (1969).

Zur Untersuchung einer möglichen Korrelation zwischen Intubationsdauer und Verletzungsgrad wurde die Schwere der Mukosaverletzungen in Anlehnung an die Bewertungsskala von Lindholm (1969) unter zusätzlicher Berücksichtigung der Intubationsdauer ausgewertet (s. Tabelle 4).

Die Auswertung der Daten aus Tabelle 4 ergab in der Gruppe I einen durchschnittlichen Verletzungsgrad von 1,7, in Gruppe II von 1,5, Gruppe III von 3,0 und in Gruppe IV einen Durchschnittsgrad von 2,125. Grundsätzlich ist zu beachten, dass aufgrund der Stärke der Gruppen I, II und IV zwischen jeweils acht und zehn beurteilbaren Patienten keine statistische Bewertung möglich ist. Insbesondere die Ergebnisse von Gruppe III haben aufgrund der Anzahl von nur drei Patienten, welche zwar jeweils denselben Verletzungsgrad aufweisen, lediglich eine geringe Aussagekraft. Insgesamt kann hieraus eine Korrelation des Schweregrades mit zunehmender Intubationsdauer nicht belegt werden. 
Lindholm (1969) konnte einen solchen Zusammenhang ebenfalls nicht feststellen. Burns et al. (1979) unterschieden Verletzungen mit intakter Mukosa und Mukosa durchdringende Verletzungen. Es ergab sich eine Zunahme der Häufigkeit mukosaler Schäden mit steigender Intubationsdauer, jedoch gab es ebenfalls keine Korrelation mit dem Schweregrad.

Colice et al. (1989), Benjamin und Holinger (2008) sowie Colton House et al. (2011) stellten ebenfalls keinen Einfluss der Intubationsdauer auf den Schweregrad der Verletzung fest.

Zur Beurteilung der Schwere der Mukosaverletzung sind die Abheilungsdauer und gegebenenfalls resultierende Stenosen wichtige Aspekte. Da lediglich ein Patient für die vorgesehene Nachuntersuchung zur Verfügung stand, können über Abheilungstendenz und Spätfolgen der Mukosaverletzungen keine Aussagen gemacht werden.

Bei vier Patienten fanden sich Hämatome der Stimmlippen. In der Literatur werden Hämatome als Intubationstraumata beschrieben, meist linksseitig aufgrund der rechten Führungshand bei der Intubation (Reber et al. 2007). In der vorliegenden Untersuchung zeigten sich zweimal rechtsseitige Hämatome, in einem Fall beidseitig. Nur ein Hämatom war linksseitig. Bei der Intubation wurden von den Ärzten keine Sichteinschränkungen oder Intubationsschwierigkeiten dokumentiert. Dennoch bleibt eine Verletzung während der Intubation offenbar möglich. Trotz kontinuierlicher Tubusfixation muss ein Trauma nach der Intubation durch Bewegung und Rotation ebenfalls als mögliche Ursache in Betracht gezogen werden.

In der heutigen Intensivmedizin sind Tuben aus thermosensiblen PCV-Materialien Standard. Ebenso beinhaltet das moderne Atemwegsmanagement zum einen die Vermeidung unnötiger Tubusbewegungen im Larynx, und zum anderen eine regelmäßige Kontrolle des Cuffdrucks. Daher ist es bemerkenswert, dass die Inzidenz der Schleimhautverletzungen in dieser Studie nicht geringer ist als in den früheren Untersuchungen von Lindholm (1969) und Burns et al. (1979), obgleich diese auf einem älteren Stand der Verfahrenstechnik basieren.

\subsection{Bewegungsstörungen der Stimmlippen}

Rekurrensparesen nach Intubation sind ein wiederholt beschriebenes und bisher nicht ausführlich untersuchtes Phänomen. Dieses bezieht sich jedoch meist auf kurzzeitige 
Intubationen. Insbesondere auch deshalb sind in dieser Studie langzeitintubierte Patienten untersucht worden.

Die Häufigkeit intubationsbedingter laryngealer Bewegungsstörungen wird in der Literatur sehr unterschiedlich angegeben. Friedrich et al. (2000) untersuchten 210 Patienten prä- und postoperativ und fanden bei drei Patienten $(1,4 \%)$ Bewegungsstörungen der Stimmlippen. Bei Benninger et al. (1998) betrug der Anteil intubationsbedingter Rekurrensparesen mit einseitigen Bewegungsstörungen 7,5\% (21 von 280 untersuchten Patienten) und mit beidseitigen Bewegungsstörungen 15,4 \% (18 von 117 Patienten). Diese Inzidenzen bezogen sich jedoch auf Kurzzeitintubationen mit einer Intubationsdauer unter 24 Stunden.

Bewegungsstörungen nach Langzeitintubation sind bisher vorwiegend als Einzelfallbeschreibungen zu finden (Cheong et al. 1994; Dalton 1995; Nuutinen und Kärja 1981; Ono et al. 2000; Via-Reque und Rattenborg 1981; Vogelhut und Downs 1979) und kaum systematisch untersucht.

In der vorliegenden Untersuchung zeigten 14 der 31 Patienten (45\%) Bewegungsstörungen der Stimmlippen nach einer Intubationszeit von mehr als 24 Stunden. Fünf dieser Patienten (36 \%) hatten beidseitige Bewegungsstörungen, die übrigen neun (64\%) wiesen einseitige Mobilitätseinschränkungen auf (s. Tabelle 5).

Bei der Beurteilung der Befunde muss berücksichtigt werden, dass neun der Patienten mit Mobilitätsstörungen kardio-thorakal operiert wurden und damit der Schädigungsmechanismus nicht sicher auf die Intubation zurückzuführen ist. Im Rahmen solcher Eingriffe ist eine direkte Schädigung des N. recurrens aufgrund des anatomischen Verlaufes durch eine Sternotomie (Hamdan et al. 2002) oder durch eine direkte Manipulation an der Aorta möglich (Ishimoto et al. 2002). Dabei wird mehrheitlich der linke N. recurrens verletzt (Itagaki et al. 2007; Shafei et al. 1997). Die Häufigkeit solcher Verletzungen wird in der Literatur mit 2,3\% angegeben und betrifft insbesondere Eingriffe in Nähe oder an der Aorta (Itagaki et al. 2007).

Von den Patienten mit Bewegungsstörungen nach stattgehabtem endothorakalen Eingriff wiesen vier Patienten beidseitige, vier Patienten nur eine linksseitige und ein Patient eine rechtsseitige Bewegungsstörung auf.

Bei der Anlage eines zentralvenösen Katheters kann es ebenso zu einer Nervenläsion kommen. Vornehmlich werden hiernach Bewegungsstörungen der rechten Stimmlippe beschrieben (Sim und Robertson 1989). 
Eine intraoperative Nervenreizung oder -verletzung muss bei den Patienten der vorliegenden Studie, bei denen eine Mobilitätsstörung auftrat, somit als möglicher Schädigungsmechanismus ebenfalls in Betracht gezogen werden. Die Häufigkeit wäre jedoch im Vergleich zur bestehenden Literatur deutlich erhöht. Weitere Schädigungsmechanismen, einschließlich der Intubation, sind somit in Betracht zu ziehen.

Ferner bestand bei keinem Patienten ein laryngoskopischer Vorbefund, so dass vorbestehende asymptomatische Paresen anderer Ursache nicht auszuschließen sind. Deren Inzidenz beträgt in der Studie von Friedrich et al. (2000) 1,9\%.

Bei den Patienten Nr. 28 und Nr. 29 mit einseitigen Stimmlippenlähmungen (s. Tabelle 3) führte eine respiratorische Insuffizienz bei einem Zustand nach Hirnstammblutung sowie bei einem Zustand nach einem Krampfanfall mit intrakranieller Blutung zur Intubation. Bei Patient Nr. 28 bleibt eine Läsion des X. Hirnnervenkerns im Rahmen der Hirnstammblutung differentialdiagnostisch möglich. Bei Patient Nr. 29 lag jedoch eine periphere Lähmung vor, die auch bei der Untersuchung nach Extubation keinen Hinweis auf eine zentrale Genese zuließ.

Bei sechs Patienten des vorliegenden Kollektivs fanden sich laryngeale Bewegungsstörungen, obwohl kein oder allenfalls ein aortenferner endothorakaler Eingriff erfolgt war. Hier bleibt als möglicher Schädigungsmechanismus ein zu hoch sitzender Tubuscuff mit konsekutiver Druckbelastung des Ramus anterior des N. laryngeus recurrens zwischen eingedrücktem paraglottischem Raum und Schildknorpelplatte bestehen (s. Abbildung 2).

Colton House et al. (2011) kamen in Ihrer prospektiven Studie laryngealer Befunde von Patienten mit einer Mindestintubationsdauer von $48 \mathrm{~h}$ zu einem vergleichbaren Ergebnis. Der Schwerpunkt deren Untersuchung lag auf der Betrachtung einer Korrelation laryngealer Verletzungen mit der Intubationsdauer unter Berücksichtigung der Tubusgröße, Alter, Geschlecht, Gewicht und Größe der Patienten. Bewegungsstörungen der Stimmlippen wurden mit unter den laryngealen Verletzungen zusammengefasst. Sie detektierten bei 24 der 61 untersuchten Patienten (39\%) Bewegungsstörungen. Mit $54 \%$ waren dabei die einseitigen Bewegungsstörungen (li. $n=12$, re. $n=1$ ) häufiger als die beidseitigen. Die linksseitige Stimmlippe war wie auch in der vorliegenden Untersuchung führend betroffen (s. Tabelle 5). Der Grund hierfür ist bisher nicht weiterführend untersucht. Ein denkbarer Schädigungsmechanismus ist die meist rechtsseitige orale Tubusausleitung. 
Patienten mit stattgehabten kardio-thorakalen Eingriffen, Hals- oder Schilddrüseneingriffen sowie vorbekannter Heiserkeit wurden von der Studie von Colton House et al. (2011) im Vorfeld ausgeschlossen. Die dokumentierten Bewegungsstörungen wurden auf die Intubation zurückgeführt.

Nach Auswertung der in Tabelle 5 dargestellten Bewegungsstörungen der Stimmlippen kann in der vorliegenden Studie keine Korrelation zwischen der Häufigkeit von Bewegungsstörungen der Stimmlippen und einer steigenden Intubationsdauer nachgewiesen werden. Bemerkenswert ist jedoch, dass die in dieser Studie festgestellte Inzidenz von Bewegungsstörungen nach Langzeitintubation im Vergleich zu den zu vorgestellten Literaturdaten zur Kurzzeitintubation deutlich höher ausfällt.

Des Weiteren wurde ein möglicher Zusammenhang zwischen Schweregrad der entstandenen Bewegungsstörungen und Dauer der Intubation untersucht. Unter Schweregrad wurden das Ausmaß der Bewegungsstörung und die Persistenz der Symptomatik zusammengefasst. Eine beidseitige Bewegungseinschränkung wurde schwerwiegender eingestuft als eine einseitige.

Es kann im Zuge dieser Studie nicht festgestellt werden, dass die Schwere der Bewegungsstörungen mit der Dauer der Intubation ansteigt (s. Tabelle 5).

Bei den Patienten mit dokumentierten Bewegungsstörungen wurde der jeweilige Grad der Schleimhautläsionen ergänzend betrachtet. Ein Zusammenhang zwischen Ausmaß der Schleimhautläsionen und den dokumentierten Bewegungsstörungen ist ebenfalls nicht feststellbar.

Die Persistenz der Lähmungen ist im Zuge der Einstufung des Schweregrades der beobachteten Mobilitätsstörungen ebenfalls zu berücksichtigen. Über eine Persistenz der Lähmungen kann jedoch in dieser Untersuchung keine Aussage gemacht werden, da lediglich ein Patient für die geplante Nachuntersuchung zur Verfügung stand.

Bei diesem Patienten wurden initial beidseitige Mobilitätsstörungen mit linksseitiger Stimmbandlähmung und rechtsseitiger Abduktionsstörung (Pat.Nr. 17) dokumentiert. Ein Jahr nach Extubation zeigte sich ein Normalbefund.

Whited (1979) präsentierte Ergebnisse von 16 Patienten mit Stimmlippenbewegungsstörungen nach einer Intubationsdauer von mindestens fünf Tagen. Bei sämtlichen Patienten kam es bereits innerhalb von 16 Wochen zu einer kompletten Rückbildung der Bewegungseinschränkung. 


\subsection{Trachealläsionen}

Bei 13 der 31 untersuchten Patienten konnte eine Tracheoskopie durchgeführt werden. Bei vier dieser Patienten war die Aufnahmequalität mäßig und somit die Beurteilung nur eingeschränkt möglich. Die Untersuchungstiefe war ebenfalls bei sämtlichen Patienten unterschiedlich.

Es bestand daher keine gemeinsame Auswertungsgrundlage für eine vergleichende Darstellung der Befunde. Die trachealen Befunde wurden als nebenbefundliche Einzelfallbeschreibungen bewertet.

\subsection{Ausblick}

Eine Korrelation zwischen Intubationsdauer und Häufigkeit sowie Schweregrad auftretender Larynxverletzungen und Bewegungsstörungen kann in der vorliegenden Untersuchung nicht belegt werden.

Jedoch ist in dieser Studie festzustellen, dass die Inzidenz von Bewegungsstörungen der Stimmlippen nach Langzeitintubation mit $45 \%$ (14 von 31 Patienten) höher ausfällt als in Ergebnissen vergleichbarer Untersuchungen nach Kurzzeitintubation. Die jeweils darin ermittelten Häufigkeiten divergieren zwar stark, sind jedoch insgesamt deutlich geringer als der obige Wert (Friedrich et al. (2000): 1,4 \%, n=210; Benninger et al. (1998): 7,5 \% einseitige Bewegungsstörungen, $\mathrm{n}=280 ; 15,4 \%$ beidseitige Bewegungsstörungen, $\mathrm{n}=117$ Patienten).

In der modernen Intensivmedizin sind thermosensible PVC-Materialien der Tuben Standard. Ebenso werden im modernen Atemwegsmanagement Bewegungen des Tubus im Larynx vermieden und eine regelmäßige Kontrolle des Cuffdrucks standardisiert durchgeführt. Dennoch ist die Inzidenz der Schleimhautverletzungen in dieser Untersuchung nicht geringer als in früheren Studien. Sämtliche Patienten, bei denen die Larynxschleimhaut dokumentiert werden konnte (29 von 31 Patienten), wiesen entsprechende Verletzungen auf. Die Lokalisationen der Larynxverletzungen gehen mit den Ergebnissen bestehender Untersuchungen konform.

Die Inzidenz der laryngealen Verletzungen und insbesondere der Bewegungsstörung in der vorliegenden Studie legen die Notwendigkeit von Präventionsmaßnahmen nahe. Eine Laryngoskopie nach Extubation langzeitintubierter Patienten erscheint zur Festlegung erforderlicher Therapien empfehlenswert. Somit können Komplikationen, wie 
beispielweise eine Aspirationspneumonie, reduziert und eine Verbesserung des Behandlungsergebnisses dieser Risikopatientengruppe erreicht werden. 


\section{Zusammenfassung}

Ziel dieser Arbeit ist die Darstellung der Häufigkeit und des Schweregrades auftretender Bewegungsstörungen der Stimmlippen und laryngealer Verletzungen nach Langzeitintubation.

Hierzu erfolgte bei Patienten mit einer Mindestintubationsdauer von 24 Stunden innerhalb der ersten 24 Stunden nach Extubation eine flexible Tracheo-Laryngoskopie.

Eine Korrelation zwischen Intubationsdauer und Häufigkeit sowie Schweregrad auftretender Larynxverletzungen und Bewegungsstörungen kann in der vorliegenden Untersuchung nicht festgestellt werden.

Die Inzidenz der Mobilitätsstörungen nach Langzeitintubation ist jedoch deutlich höher als nach Kurzzeitintubationen. In der vorliegenden Untersuchung wurden bei $45 \%$ der Patienten (14 von 31 Patienten) Bewegungsstörungen der Stimmlippen dokumentiert. Die Inzidenzen aus Arbeiten zu Kurzzeitintubation sind unterschiedlich, aber deutlich geringer.

Als mögliche Ursache der Bewegungsstörungen ist ein zu hoch sitzender Tubuscuff mit konsekutiver Druckbelastung des Ramus anterior des N. laryngeus recurrens zwischen eingedrücktem paraglottischen Raum und Schildknorpelplatte zu diskutieren.

In der Ergebnisbetrachtung dieser Studie muss dabei kritisch berücksichtigt werden, dass neun der Patienten mit Bewegungsstörungen im Vorfeld der Untersuchung kardiothorakal operiert wurden. Der Schädigungsmechanismus kann hier nicht zweifelsfrei auf die Intubation zurückgeführt werden. Eine intraoperative Verletzung oder Schädigung des Nerven bleibt möglich. Die Häufigkeit wäre im Verglich zu bestehender Literatur jedoch deutlich erhöht.

Trotz moderner Materialien und einer atemwegsschonenden Verfahrenstechnik in der heutigen Intensivmedizin ist die Inzidenz der Schleimhautverletzungen in dieser Untersuchung nicht geringer als in früheren Studien.

Die Larynxschleimhaut sämtlicher Patienten, bei denen eine Dokumentation gelang (29 von 31 Patienten), weist entsprechende Verletzungen auf. Die Lokalisationen der Larynxverletzungen stimmen mit den Ergebnissen bestehender Untersuchungen überein.

Das Wissen um die Wahrscheinlichkeit auftretender laryngealer Verletzungen und insbesondere von Bewegungsstörungen der Stimmlippen nach Langzeitintubation ist für 
die Planung von Präventionsmaßnahmen bedeutsam. Um nachfolgend die Notwendigkeit von Therapiemaßnahmen zu bestimmen, erscheint anhand der vorliegenden Ergebnisse eine endoskopische Kontrolle des Larynx und der Stimmlippenbeweglichkeit nach Extubation empfehlenswert. Die frühzeitige Einleitung erforderlicher Therapien kann zur Reduktion von Komplikationen, wie beispielsweise einer Aspirationspneumonie, beitragen und somit zu einer Verbesserung des Behandlungsergebnisses dieser Risikopatientengruppe führen. 


\section{Literaturverzeichnis}

AROLD R (1981): Chirurgie des Nervus recurrens. Untersuchung zum Problem der Funktionswiederherstellung nach Rekurrensverletzung. Fortschr Med 99: $1612-4$

Baraka A, Hemady K, Yamut F, Yazigi W, Canalis RF (1981): Postoperative paralysis of phrenic and recurrent larygal nerves. Anesthesiology 55, 78-80

BAUER H (1958): Fehler und Gefahren. Zur Dehnungsverletzung der Nervus recurrens durch Intubation bei Narkose. Anaesthesist $\underline{7}, 173-175$

BENJAMIN B, HoLinger LD (2008): Laryngeal Complications of Endotracheal Intubation. Ann Otol Rhinol Laryngol Laryngology 117, 2-20

BENNINGER MS, GILLEN JB, ALtMANN JS (1998): Changing etiology of vocal fold immobility. Laryngoscope $\underline{108}$, 1346-1350

BISHOP MJ, WEYMULLER EA, FINK BR (1984): Laryngeal effects of prolonged intubation. Anesth Analg $\underline{63}, 335-342$

BRANDT L (1986): Die Geschichte der Intubationsnarkose unter besonderer Berücksichtigung der Entwicklung des Endotrachealtubus. Anaesthesist 35, 523-530

BRANDWEIN M, ABRAMSON AL, SHIKOWSKI MJ (1986): Bilateral vocal cord paralysis following endotracheal intubation. Arch Otolaryngol Head Neck Surg 112, $877-882$

BRAUSE H, FRIEDRICH P: Grundlagen der Intensivmedizin. In: Schulte am Esch J, Bause H, Kochs E, Scholz J, Standl T (Hrsg.): Duale Reihe AnästhesieIntensivmedizin, Notfallmedizin, Schmerztherapie. 4. Auflage, Thieme, Stuttgart 2011, 405-523

Burns HP, DAYAL VS, SCOTT A, VAN Nostrand AW, BRYCE DP (1979):

Laryngotracheal trauma: Observations on its pathogenesis and its prevention following prolonged orotracheal intubation in the adult. Laryngoscope $\underline{89}$, 1316-1325 
CAvo JW (1985): True vocal cord paralysis following Intubation. Laryngoscope $\underline{95}$, $1352-1359$

CheOng KF, ChAn MY, Sin-FAI-LAM KN (1994): Bilateral vocal cord paralysis following endotracheal intubation. Anaesth Intensive Care 22, 206-208

COLICE GL (1992): Resolution of laryngeal injury following translaryngeal intubation. The American Review of Respiratory Disease 145, 361-364

Colice GL, STUKel TA, DAin B (1989): Laryngeal complications of prolonged intubation. Chest $\underline{96}, 877-884$

Colton House J, NoOrdziJ JP, Murgia B, LANGMOre S (2011): Laryngeal injury from prolonged intubation: A Prospective Analysis of Contributing Factors. Laryngoscope $\underline{121}, 596-600$

COOPER JD, GRILlo HC (1972): Analysis of problems related to cuffs on intratracheal tubes. Chest $\underline{62,21 S-27 \mathrm{~S}}$

COX R, WeLboRn S (1981): Vocal cord paralysis after endotracheal anesthesia. South Med J $\underline{74}, 1258-1259$

DALTON C (1995): Bilateral vocal cord paralysis following endotracheal intubation. Anaesth Intensive Care 23, 350-351

Ding LW, WANG HC, Wu HD, Chang CJ, YANG PC (2006): Laryngeal ultrasound: a useful method in predicting post-extubation stridor. A pilot study. Eur Respir J 27, 384-389

Dralle H, Kruse E, Hamelmann W, Grond S, Neumann H, Sekulla C, Richter C, Thomusch O, MÜHLig H-P, Voß J, Timmermann W (2004): Nicht jeder Stimmlippenstillstand nach Schilddrüsenoperation ist eine chirurgischbedingte Rekurrensparese. Stellungnahme der „Interdisziplinären Studiengruppe Intraoperatives Neuromonitoring Schilddrüsenchirurgie“ zur Problematik intubationsbedingter Rekurrensparesen. Chirurg $\underline{75}$, 810-822

ECKEL HE, SiTTEL C (2001): Beidseitige Rekurrenslähmungen. HNO $\underline{49}$, 166-179 
ELLIS PDM, BENNETT J (1977): Laryngeal trauma after prolonged endotracheal intubation. J Laryngol Otol 91, 69-74

ELLIS PDM, PALLISTER WK (1975): Recurrent laryngeal nerve palsy and endotracheal intubation. J Laryngol Otol $\underline{89}$, 823-826

ENDO K, OKABE Y, MARUYAMA Y, TSUKATANi T, FURUKAWA M (2007): Bilateral vocal cord paralysis caused by laryngeal mask airway. Am J Otolaryngol $\underline{28}$, 126-129

FREEMAN BD, BORECKI IB, COOPERSMITH CM, BUCHMAN TG (2005): Relationship between tracheostomy timing and duration of mechanical ventilation in critically ill patients. Crit Care Med $\underline{33}$, 2513-2520

FRIEDRICH T (2002): Schlusswort zur Arbeit „Die Rekurrensparese als Intubationsschaden?“' Chirurg $\underline{73}, 539-544$

FrIEDRICH T, HÄNSCH U, EICHFELd U, STEINERT M, STAEMMLER A, SCHÖNFELDER M (2000): Die Recurrensparese als Intubationsschaden? Chirurg 71, 539-544

Goerig M, Brandt L (1986): Die Geschichte der Tracheotomie. Teil II. Anaesthesist $\underline{35}, 397-402$

GRIFFITHS J, BARBER VS, MORGAN L, YOUNG JD (2005): Systematic review and metaanalysis of studies of the timing of tracheostomy in adult patients undergoing artificial ventilation. BMJ 330, 1243

HAHN F, MARTIN JT, LILLIE JC (1970): Vocal-Cord paralysis with endotracheal

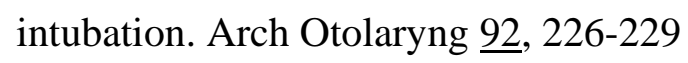

HAMDAN AL, MOUKARBEl RV, FARHAT F, OBEID M (2002): Vocal cord paralysis after open-heart surgery. Eur J Cardiothorac Surg 21, 671-674

HARRISON GA, TONKIN JP (1968): Prolonged (therapeutic) endotracheal intubation. Br J Anaesth $\underline{40}, 241-249$

Heinemann M, KalfF G (1982): Mögliche Mechanismen, Differentialdiagnosen und Prophylaxe bei Stimmlippenlähmungen nach endotrachealer Intubation. Anaesthesist $\underline{31}, 353-358$ 
Ishimoto SI, Ito K, Toyama M, KAwASE I, Kondo K, OSHIMA K, NiImi S (2002): Vocal cord paralysis after surgery for thoracic aortic aneurysm. Chest $\underline{121}, 1911-1915$

ITAGAKI T, KIKURA M, SATO S (2007): Incidence and risk factors of postoperative vocal cord paralysis in 987 patients after cardiovascular surgery. Ann Thorac Surg $\underline{83}, 2147-2152$

KEANE WM, DENNENEY JC, Rowe LD, ATKIns JP (1982): Complications of intubation. Ann Otol Rhinol Laryngol 91, 584-587

KLOSE R, KÖNIG W, DreisZ I, LuTZ H (1978): Allgemeine Aspekte zur Wahl von Langzeitintubation und Tracheotomie. Prakt Anästh 13, 249-260.

Kroll DA, Caplan RA, Posner K, Ward RJ, Cheney FW (1990): Nerve injury associated with anesthesia. Anesthesiology 73, 202-207

Kruse E, Olthoff A, SchiEl R (2006): Functional anatomy of the recurrent and superior laryngeal nerve. Langenbecks Arch Surg $\underline{391}$, 4-8

LARSEN R: Anästhesie. 10. Auflage; Urban \&Fischer in Elsevier, München 2013

Lemburg P, Müntefering H, Stemann EA (1971): Pathologisch- anatomische Befunde am Larynx nach Langzeitintubation. Monatsschr Kinderheilkd 119, 375-379

LINDHOLM CE (1969): Prolonged endotracheal intubation. Acta Anaesthesiol Scand Suppl $\underline{33}, 1-131$

LU YH, HSIEH MW, TONG YH (1999): Unilateral vocal cord paralysis following endotracheal intubation - a case report. Acta Anaesthesiol Sin 37, 221-224

LUCKHAUPT H, BRUSIS T (1986): Zur Geschichte der Intubation. Laryngo Rhino Otol $\underline{65}, 506-510$

MAntel K, Westhues G (1967): Tracheotomie oder Langzeit-Intubation bei der akuten Ateminsuffizienz? Trans Am Neurol Assoc 92, 906-908 
Messingschlager W (1970): Trachealstenosen nach Langzeitintubation. Arch Klin Exp Ohren Nasen Kehlkopfheilkd 196, 337-341

MiNUCK M (1976): Unilateral vocal-cord paralysis following endotracheal intubation. Anaesthesiology $\underline{45}, 448-449$

NUUTINEN J, KÄRJÄ J (1981): Bilateral vocal cord paralysis following general anaesthesia. Laryngoscope 91, 83-86

Olthoff A, SchiEl R, KRUSE E (2003): Beidseitige Stimmlippenlähmung nach Intubationsnarkose. Vortrag auf der 20. wissenschaftlichen Jahrestagung der DGPP, Rostock 12.-14.09.2003, German Medical Science 2003. DocV11

ONO S, NiSHIYAMA T, HANAOKA K (2000): Hoarseness after endotracheal intubation caused by submucosal hemorrage of the vocal cord and recurrent nerve palsy. Masui $\underline{49}, 881-883$

Pfannenstiel TJ, Gal TJ, Hayes DK, Myers KV (2007): Vocal fold immobility following burn intensive care. Otolaryngol Head Neck Surg 137, 152-156

Probst R, Grevers G, Iro H: Hals-Nasen-Ohren-Heilkunde. 3. Auflage; Thieme, Stuttgart 2008

Prometheus Atlas der Anatomie: Kopf, Hals und Neuroanatomie. Hrsg:v. Schünke M, Schulte E, Schumacher U, Voll M, Wesker KH. 3.Auflage; Thieme, Stuttgart 2012

Reber A, HAuenstein L, ECHTERnACH M (2007): Pharyngolaryngeale Beschwerden nach Allgemeinanästhesien. Anästhesiologische und laryngologische Aspekte. Anaesthesist 56, 177-192

ROTH F, NEIGER M, TSCHIRREN B (1967): Erfahrungen in der nasotrachealen Langzeit-Intubation. Pract Otorhinolaryngol (Basel) 29, 385-396

SCHIEBLER TH, SchmidT W, ZiLles K: Anatomie. 8. Auflage; Springer Berlin 1999 
Schiel R, Olthoff A, KRUSE E (2004): Untersuchungen zur Anatomie des Ramus posterior des Nervus recurrens und seiner Beziehung zum Musculus interarytaenoideus. Vortrag auf der 21. wissenschaftlichen Jahrestagung der DGPP, Freiburg/Breisgau 10.-12.09.2004 German Medical Science 2004, DocV02

SChUlte HK, SEIDNER W: Stimme- Physiologische Grundlagen. In: Wendler J, Seidner W, Eysholdt U (Hrsg.): Lehrbuch der Phoniatrie und Pädaudiologie. 4. Auflage; Thieme, Stuttgart 2005, 71-90

SEIDNER W, EyshOLDT U: Stimme- Diagnostik. In Wendler J, Seidner W, Eysholdt U (Hrsg.): Lehrbuch der Phoniatrie und Pädaudiologie. 4. Auflage; Thieme, Stuttgart 2005, 105-138

Shafei H, El-Kholy A, Azmy S, Ebrahim M, Al-Ebrahim K (1997): Vocal cord dysfunction after cardiac surgery: an overlooked complication. Eur J Cardiothorac Surg 22, 564-566

SIM DW, RoBERTSON MR (1989): Right vocal paralysis after internal jugular vein cannulation. J Laryngol Otol $\underline{103}, 424$

SовоттA Atlas der Anatomie des Menschen. Hrsg. v. Waschke J, Böckers TM, Paulsen F. 23. Auflage; Urban \&Fischer in Elsevier, München 2010

Stauffer Jl, Olson DE, PetTy Tl (1981): Complications and consequences of endotracheal intubation and tracheotomy. A prospective study of 150 critically ill adult patients. Am J Med $\underline{70}$, 65-76.

STENQVist O, BAGGE U (1979): Cuff pressure and microvascular occlusion in the tracheal mucosa. An intravital microscopic study in the rabbit. Acta Otolaryngol $\underline{88}, 451-454$

Stenqvist O, BAgGE U, NiLsSON K (1979): The tracheal mucosa microvasculature and microcirculation. Intravital microscopic observations in rabbits and a histologic study in man. Acta Otolaryngol ㄱ, 123-128

SUNDERLAND S, SWANEY WE (1952): The intraneural topography of the reccurent laryngeal nerv in man. Anat Rec 114, 411-426 
Via-Reque E, RAtTenborg CC (1981): Prolonged oro- or nasotracheal intubation. Crit Care Med $\underline{9}, 637-639$

Vila J, Bosque MD, García M, Palomar M, Quesada P, RAmis B (1997):

Endoscopic evolution of laryngeal injuries caused by translaryngeal intubation. Eur Arch Otorhinolaryngol 254 Suppl 1, S97-100

Vogelhut MM, Downs JB (1979): Prolonged endotracheal intubation. Chest $\underline{76}$, $110-111$

WASON R, GUPTA P, GOGIA AR (2004): Bilateral adductor cord paresis following endotracheal intubation for general anaesthesia. Anaesth Intensive Care $\underline{32}, 417-418$

WENDLER J, SEIDNER W: Stimme- Klinik. In: Wendler J, Seidner W, Eysholdt u (Hrsg.): Lehrbuch der Phoniatrie und Pädaudiologie. 4. Auflage; Thieme, Stuttgart 2005, 139-203

Weymuller EA, Bishop MJ, Fink BR, Hibbard AW, Spelman FA (1983):

Quantification of intralaryngeal pressure exerted by endotracheal tubes. Ann Otol Rhinol Laryngol 92, 444-447

WHITED RE (1979): Laryngal dysfunction following prolonged intubation. Ann Otol Rhinnol $\underline{88}, 474-478$

YOUNG N, STEWARD S (1953): Laryngeal lesions following endotracheal anaesthesia: a report of twelve adult cases. Br J Anaesth 25, 32-42

Zumtobel M, End A, BigenZahn W, KlepetKo W, Schneider B (2006): Beeinträchtigung der Lebensqualität bei Patienten mit einseitiger Rekurrensparese nach thoraxchirurgischen Eingriffen. Chirurg 77, 518-522 


\section{Danksagung}

An erster Stelle gilt mein Dank Prof. Dr. Arno Olthoff für die Übernahme der wissenschaftlichen Betreuung dieser Dissertation. Insbesondere möchte ich mich für die Anregungen und Ratschläge sowie für die fachliche Unterstützung in den klinischen Untersuchungen zu dieser Studie bedanken.

Ebenfalls sehr herzlich danke ich dem gesamten Personal der Intensivstationen des Klinikums Göttingen für seine Unterstützung im Zuge der durchgeführten Untersuchungen.

Mein besonderer Dank gilt meinen Eltern für Ihren Rückhalt und Zuspruch, Dr. Esther Kindler für die stete Inspiration und Motivation, Marc Hilgert für die wertvolle Unterstützung bei der Text-, Bild- und Datenverarbeitung sowie Dr. Lars Gonschorek für das gewissenhafte Lektorat. 